\title{
New Isatin-Indole Conjugates: Synthesis, Characterization, and a Plausible Mechanism of Their in vitro Antiproliferative Activity
}

This article was published in the following Dove Press journal: Drug Design, Development and Therapy

\author{
Reem I Al-Wabli (D' \\ Aliyah A Almomen (D)' \\ Maha S Almutairi' \\ Adam B Keeton ${ }^{2}$ \\ Gary A Piazza ${ }^{2}$ \\ Mohamed I Attia (D) ${ }^{1,3}$ \\ 'Department of Pharmaceutical \\ Chemistry, College of Pharmacy, King \\ Saud University, Riyadh I I45I, Saudi \\ Arabia; ${ }^{2}$ Department of Oncologic \\ Sciences and Pharmacology, Drug \\ Discovery Research Center, Mitchell \\ Cancer Institute, University of South \\ Alabama, Mobile, AL 36604-I405, USA; \\ ${ }^{3}$ Medicinal and Pharmaceutical Chemistry \\ Department, Pharmaceutical and Drug \\ Industries Research Division, National \\ Research Centre (ID: 600I46I8), Giza \\ 12622, Egypt
}

Background: Cancer remains the leading cause of human morbidity universally. Hence, we sought to assess the in vitro antiproliferative activity of new isatin-based conjugates (5a-s) against three human cancer cell lines.

Methods: The antiproliferative activities of compounds $\mathbf{5 a}-\mathbf{s}$ were evaluated in vitro and their ADME (absorption, distribution, metabolism and excretion) was carried out using standard protocols. Subsequently, Western blot analysis was conducted to elucidate the potential antiproliferative mechanism of compounds $\mathbf{5 a}-\mathbf{s}$.

Results: The in vitro antiproliferative activities of compounds $\mathbf{5 a}-\mathbf{s}$ against the tested cancer cell lines ranged from 20.3 to $95.9 \%$. Compound $\mathbf{5 m}$ had an $\mathrm{IC}_{50}$ value of $1.17 \mu \mathrm{M}$; thus, its antiproliferative potency was approximately seven-fold greater than that of sunitinib $\left(\mathrm{IC}_{50}=\right.$ $8.11 \mu \mathrm{M})$. In-depth pharmacological testing was conducted with compound $\mathbf{5 m}$ to gain insight into the potential antiproliferative mechanism of this class of compounds. Compound $\mathbf{5 m}$ caused an increase in the number of cells in the G1 phase, with a concomitant reduction of those in the G2/M and S phases. Additionally, compound 5m significantly and dose-dependently reduced the amount of phosphorylated retinoblastoma protein detected. Compound $\mathbf{5 m}$ enhanced expression of B cell translocation gene 1, cell cycle-associated proteins (cyclin B1, cyclin D1, and phosphorylated cyclin-dependent kinase 1), and a pro-apoptotic protein (Bcl-2-associated X protein gene), and activated caspase-3. ADME predictions exposed the oral liability of compounds 5a-s. Conclusion: Herein, we revealed the antiproliferative activity and ADME predictions of the newly-synthesized compounds $\mathbf{5 a}-\mathbf{s}$ and provided a detailed insight into the pharmacological profile of compound $\mathbf{5 m}$. Thus, compounds $\mathbf{5 a}-\mathbf{s}$ can potentially be exploited as new antiproliferative lead compounds for cancer chemotherapeutic.

Keywords: synthesis, isatin, indole, antiproliferative, cancer cell line

\section{Introduction}

Cancer, the second major source of mortality in humans after cardiovascular diseases, remains a considerable health obstacle worldwide. ${ }^{1}$ Although substantial advances have been made with cancer-diagnosis tools and many chemotherapeutic agents are currently available as treatments, successful management of cancer remains a medical challenge. Because current cancer-chemotherapeutic agents show a lack of selectivity and can elicit multiple-drug resistance, there exists a constant need to derive newer and safer anticancer agents. ${ }^{2-4}$

Creating novel anticancer chemotherapeutic agents based on naturally occurring bioactive substances has been gaining increased interest. ${ }^{5,6}$ 2,3-Dioxindole (isatin, I,
Correspondence: Mohamed I Attia Department of Pharmaceutical Chemistry, College of Pharmacy, King Saud University, P.O. Box. 2457, Riyadh

II45I, Saudi Arabia

Tel +966- | 46-77337

Fax+966-146-76220

Email mattia@ksu.edu.sa 
Figure 1) is a natural substance found in some plant species, which can also be present in humans, due to endogenous consumption. Compound I has a distinguished pharmacological profile and exhibits a wide spectrum of biological actions, including anti-oxidant, ${ }^{7}$ anti-inflammatory, ${ }^{8}$ antibacterial, $^{9}$ and anticancer ${ }^{10,11}$ activities. The United States Federal Drug Administration approved sunitinib and nintedanib as isatin-bearing anticancer drugs, ${ }^{10,12}$ and many compounds with an isatin scaffold such as SU6668 and SU5416 have displayed outstanding anticancer activity. ${ }^{13-15}$ Therefore, isatin-bearing drugs might exhibit extensive cytotoxicity against cancer cells.

Indole (1H-benzo[b]pyrrole, II, Figure 1$)$ has a bicyclic heterocycle scaffold that is widely distributed in animals, microbial hormones, and plants. ${ }^{16,17}$ It is considered to be a beneficial motif in drug-discovery programs because of its biodiversity, versatility, and anticancer activity. ${ }^{18-21}$ Previous evidence suggests that myriad indole-based compounds exert anticancer activities through various mechanisms, ${ }^{22-27}$ suggesting the possibility of using the indole scaffold in anticancer drugs. Notably, anticancer indole-based molecules target several pathways, such as those involving tubulin polymerization, DNA topoisomerase, histone deacetylase, proto-oncogenes, and serine/threonine (PIM) kinase. ${ }^{28-31}$

Screening the literature exposed that a number of bioactive molecules bear carbohydrazide moiety. They display a wide spectrum of biological activities including anticancer activity. $^{32-34}$

Combining the structural features of two differing pharmacophores is an effective tactic in medicinal chemistry, as new bioactive, chemotherapeutic chemical entities characterized by a reduced risk of drug-drug interactions and improved anticancer profiles can be obtained. ${ }^{35-37}$ Considering the structural characteristics of isatin and indole, merging these two bioactive moieties into a single compound through a carbohydrazide bridge is predicted to result in new promising anticancer leads. Accordingly, we aimed to synthesize and evaluate the

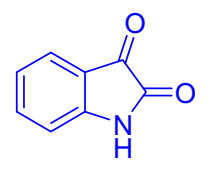

I

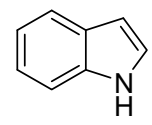

II in vitro antiproliferative activities of the molecular hybrids $\mathbf{5 a}-\mathbf{s}$. The most active antiproliferative candidate $\mathbf{5 m}$ was also examined in an in-depth pharmacological-profiling study to understand the potential mechanism whereby these compounds exert their antiproliferative effect.

\section{Materials and Methods Chemistry General}

The melting points of compounds $\mathbf{5 a}-\mathbf{s}$ were measured using the Gallenkamp melting point equipment and are presented as uncorrected values. Compounds $\mathbf{5 a}-\mathbf{s}$ were dissolved in DMSO- $d_{6}$ for nuclear magnetic resonance (NMR) measurements at 500 and $125 \mathrm{MHz}$ for ${ }^{1} \mathrm{H}$ and ${ }^{13} \mathrm{C}$, respectively, using a Bruker NMR spectrometer (Bruker, Reinstetten, Germany) at the Research Center (College of Pharmacy, King Saud University, Saudi Arabia). TMS was used as an internal standard for the NMR measurements. The measured chemical shifts are presented as $\delta$-values in parts per million (ppm). Compounds 5a-s were subjected to microanalyses at the Microanalysis Laboratory (Cairo University, Cairo, Egypt), and the results aligned well with the proposed structures (i.e., within $\pm 0.4 \%$ of the theoretical values). A 6120 LC/MS Agilent Quadrupole system with an electrosprayionization(ESI) source (Agilent Technologies, Palo Alto, CA, USA) was used to obtain the mass spectra of compounds 5a-s. Compound $\mathbf{3}$ and compounds $\mathbf{4 a}-\mathbf{n}$ were prepared as described previously. ${ }^{38}$

\section{General Procedure for Synthesizing Carbohydrazides $5 a-s$}

The acid hydrazide $3(0.18 \mathrm{~g}, 1 \mathrm{mmol})$ was dissolved in absolute ethanol $(15 \mathrm{~mL})$, and the appropriate isatin derivative $4 \mathbf{a}-\mathbf{n}(1 \mathrm{mmol})$ was added to the stirred ethanolic solution containing drops of glacial acetic acid. The reaction mixture was refluxed under continuous stirring for 4 $\mathrm{h}$, and then the hot alcoholic reaction mixture was filtered. The collected solids were re-crystallized from the ethanol/

Figure I Chemical structures of compounds I, II, and 5a-s.

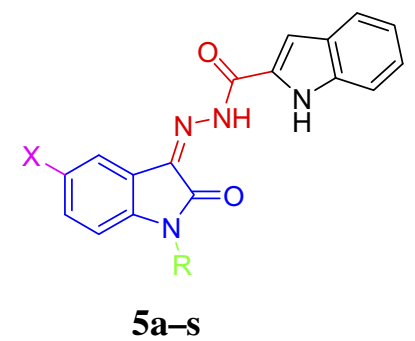


DMF mixture $(3: 1)$ to yield the corresponding target carbohydrazides $\mathbf{5 a}-\mathbf{s}$ with yields of 40 to $95 \%$.

$N^{\prime}$-[(3Z)-2-Oxo-1,2-dihydro-3H-indol-3-ylidene]-

$1 H$-indole-2-carbohydrazide (5a): Yellow powder, melting point (m.p.) $>300{ }^{\circ} \mathrm{C}$ (yield 89\%); ${ }^{1} \mathrm{H}$ NMR (DMSO-d $)_{6}$ ppm: 6.95 (d, $J=7.5 \mathrm{~Hz}, 1 \mathrm{H}, \mathrm{H}_{\mathrm{ar}}$ ), 7.09 $7.16\left(\mathrm{~m}, 2 \mathrm{H}, \mathrm{H}_{\mathrm{ar}}\right), 7.29$ (d, $\left.J=7.0 \mathrm{~Hz}, 1 \mathrm{H}, \mathrm{H}_{\mathrm{ar}}\right), 7.42$ (d, $\left.J=7.5 \mathrm{~Hz}, 1 \mathrm{H}, \mathrm{H}_{\text {ar. }}\right), 7.51\left(\mathrm{~d}, J=8.5 \mathrm{~Hz}, 1 \mathrm{H}, \mathrm{H}_{\text {ar. }}\right)$, $7.61\left(\mathrm{~s}, 1 \mathrm{H}, \mathrm{H}_{\mathrm{ar}}\right), 7.73\left(\mathrm{~d}, J=8.0 \mathrm{~Hz}, 1 \mathrm{H}, \mathrm{H}_{\mathrm{ar}}\right), 8.05$ (d, $\left.J=7.5 \mathrm{~Hz}, 1 \mathrm{H}, \mathrm{H}_{\mathrm{ar}}\right), 10.88$ (s, 1H, NH), 11.69 (s, 1H, $\mathrm{NH}), 11.97(\mathrm{~s}, 1 \mathrm{H}, \mathrm{NH}) ;{ }^{13} \mathrm{C}$ NMR $\left(\mathrm{DMSO}-d_{6}\right) \mathrm{ppm}$ : $104.2,111.1,112.9,116.1,120.7,122.3,122.7,123.2$, $125.1,127.2,127.5,129.3,133.2,137.6,144.4\left(\mathrm{C}_{\text {ar. }}\right.$, $\left.\mathrm{CH}_{\text {ar. }}, \quad \mathrm{C}=\mathrm{N}\right), \quad 160.9, \quad 165.3(2 \times \mathrm{C}=\mathrm{O}) ; \quad \mathrm{MS} \mathrm{m} / \mathrm{z}$ : $303[\mathrm{M}-\mathrm{H}]^{-}$.

$N^{\prime}$-[(3Z)-5-Bromo-2-oxo-1,2-dihydro-3H-indol-3-ylidene]-1H-indole-2-carbohydrazide $\quad(\mathbf{5 b})$ : $\quad$ Yellow powder, m.p. $>300{ }^{\circ} \mathrm{C}$ (yield 76\%); ${ }^{1} \mathrm{H} \quad \mathrm{NMR}$ (DMSO-d ${ }_{6}$ ppm: $6.90\left(\mathrm{~d}, J=8.5 \mathrm{~Hz}, 1 \mathrm{H}, \mathrm{H}_{\mathrm{ar}}\right), 7.09$ $7.14\left(\mathrm{~m}, 1 \mathrm{H}, \mathrm{H}_{\mathrm{ar}}\right), 7.28$ (d, $\left.J=7.5 \mathrm{~Hz}, 1 \mathrm{H}, \mathrm{H}_{\mathrm{ar}}\right), 7.51$ (d, $\left.J=8.0 \mathrm{~Hz}, 1 \mathrm{H}, \mathrm{H}_{\text {ar. }}\right), 7.59$ (d, $\left.J=8.0 \mathrm{~Hz}, 1 \mathrm{H}, \mathrm{H}_{\text {ar. }}\right)$, 7.65 (s, 1H, $\left.\mathrm{H}_{\text {ar. }}\right), 7.73$ (d, $\left.J=8 \mathrm{~Hz}, 1 \mathrm{H}, \mathrm{H}_{\mathrm{ar}}\right), 8.34$ (s, $\left.1 \mathrm{H}, \mathrm{H}_{\mathrm{ar}}\right), 11.01(\mathrm{~s}, 1 \mathrm{H}, \mathrm{NH}), 11.87(\mathrm{~s}, 1 \mathrm{H}, \mathrm{NH}), 11.95$ $(\mathrm{s}, 1 \mathrm{H}, \mathrm{NH}) ;{ }^{13} \mathrm{C}$ NMR (DMSO- $\left.d_{6}\right) \mathrm{ppm}: 104.8,108.6$, $112.9,113.8,117.5,120.7,121.0,122.8,125.2,127.5$, $129.2,135.2,136.4,137.7,143.5\left(\mathrm{C}_{\text {ar. }}\right.$ and $\left.\mathrm{CH}_{\text {ar. }}, \mathrm{C}=\mathrm{N}\right)$, 160.7, $164.8(2 \times \mathrm{C}=\mathrm{O}) ; \mathrm{MS} \mathrm{m} / \mathrm{z}: 381[\mathrm{M}-\mathrm{H}]^{-}, 383$ $[\mathrm{M}-\mathrm{H}+2]^{-}$.

$N^{\prime}$-[(3Z)-5-Chloro-2-oxo-1,2-dihydro-3H-indol-3-ylidene]-1H-indole-2-carbohydrazide $\quad \mathbf{( 5 c )}$ : Yellow powder, m.p. > $300{ }^{\circ} \mathrm{C}$ (yield 91\%); ${ }^{1} \mathrm{H} \quad \mathrm{NMR}$ (DMSO- $\left.d_{6}\right)$ ppm: $6.95\left(\mathrm{~d}, J=8.5 \mathrm{~Hz}, 1 \mathrm{H}, \mathrm{H}_{\mathrm{ar} .}\right), 7.09$ $7.14\left(\mathrm{~m}, 1 \mathrm{H}, \mathrm{H}_{\mathrm{ar}}\right), 7.27-7.32\left(\mathrm{~m}, 1 \mathrm{H}, \mathrm{H}_{\mathrm{ar}}\right), 7.43-7.47$ $\left(\mathrm{m}, 1 \mathrm{H}, \mathrm{H}_{\mathrm{ar}}\right), 7.51\left(\mathrm{~d}, J=8.0 \mathrm{~Hz}, 1 \mathrm{H}, \mathrm{H}_{\mathrm{ar}}\right), 7.66(\mathrm{~s}, 1 \mathrm{H}$, $\left.\mathrm{H}_{\text {ar. }}\right), 7.74\left(\mathrm{~d}, J=8.0 \mathrm{~Hz}, 1 \mathrm{H}, \mathrm{H}_{\mathrm{ar} .}\right), 8.22\left(\mathrm{~s}, 1 \mathrm{H}, \mathrm{H}_{\mathrm{ar} .}\right)$, $11.00(\mathrm{~s}, 1 \mathrm{H}, \mathrm{NH}), 11.86(\mathrm{~s}, 1 \mathrm{H}, \mathrm{NH}), 11.96(\mathrm{~s}, 1 \mathrm{H}$, $\mathrm{NH}) ;{ }^{13} \mathrm{C}$ NMR (DMSO- $d_{6}$ ) ppm:102.1, 109.3, 112.4, $112.9,117.1,120.7,122.8,125.2,126.2,126.6,127.5$, 132.4, 137.6, 138.9, $143.0\left(\mathrm{C}_{\text {ar. }}\right.$ and $\left.\mathrm{CH}_{\text {ar. }}, \mathrm{C}=\mathrm{N}\right), 163.5$, $165.1(2 \times \mathrm{C}=\mathrm{O}) ; \mathrm{MS} \mathrm{m} / \mathrm{z}: 337[\mathrm{M}-\mathrm{H}]^{-} ; 339[\mathrm{M}-\mathrm{H}+2]^{-}$.

$N^{\prime}$-[(3Z)-5-Fluoro-2-oxo-1,2-dihydro-3H-indol-3-ylidene]-1 $H$-indole-2-carbohydrazide $\quad(\mathbf{5 d})$ : Yellow powder, m.p. $>300{ }^{\circ} \mathrm{C}$ (yield 95\%); ${ }^{1} \mathrm{H}$ NMR (DMSO-d $)_{6}$ ppm: $6.93\left(\mathrm{dd}, J=4,8.5 \mathrm{~Hz}, 1 \mathrm{H}, \mathrm{H}_{\text {ar. }}\right.$ ), $7.10\left(\mathrm{~d}, J=8.0 \mathrm{~Hz}, 1 \mathrm{H}, \mathrm{H}_{\mathrm{ar}}\right), 7.25-7.30\left(\mathrm{~m}, 2 \mathrm{H}, \mathrm{H}_{\mathrm{ar}}\right)$, $7.50\left(\mathrm{~d}, J=8.5 \mathrm{~Hz}, 1 \mathrm{H}, \mathrm{H}_{\mathrm{ar}}\right), 7.66\left(\mathrm{~s}, 1 \mathrm{H}, \mathrm{H}_{\mathrm{ar}}\right), 7.74$ (d, $\left.J=8.0 \mathrm{~Hz}, 1 \mathrm{H}, \mathrm{H}_{\text {ar. }}\right), 8.02\left(\mathrm{~d}, J=8.0 \mathrm{~Hz}, 1 \mathrm{H}, \mathrm{H}_{\text {ar. }}\right)$, 10.89 (s, 1H, NH), 11.79 (s, 1H, NH), 11.95 (s, 1H,
$\mathrm{NH}) ;{ }^{13} \mathrm{C}$ NMR (DMSO-d $)$ ppm: 108.4, 111.8, 112.8, $112.9,113.1,114.3,120.7,120.9,122.7,122.8,125.3$, $125.5,137.5,139.1\left(\mathrm{C}_{\text {ar. }}, \mathrm{CH}_{\text {ar. }}, \mathrm{C}=\mathrm{N}\right), 157.9\left(\mathrm{C}_{1^{\prime}-\mathrm{F}}, J=\right.$ $\left.235.0 \mathrm{~Hz}, \mathrm{C}_{\text {ar. }}\right), 163.7,165.4(2 \times \mathrm{C}=\mathrm{O}) ; \mathrm{MS} \mathrm{m} / \mathrm{z}$ : $321[\mathrm{M}-\mathrm{H}]^{-}$.

$N^{\prime}$-[(3Z)-5-Methoxy-2-oxo-1,2-dihydro-3H-indol-3-ylidene]-1 $H$-indole-2-carbohydrazide (5e): Orange powder, $\mathrm{m}$. p. $>300{ }^{\circ} \mathrm{C}$ (yield $75 \%$ ); ${ }^{1} \mathrm{H}$ NMR (DMSO- $d_{6}$ ) ppm: $3.82(\mathrm{~s}$, $\left.3 \mathrm{H}, \mathrm{OCH}_{3}\right), 6.86\left(\mathrm{~d}, J=8.5 \mathrm{~Hz}, 1 \mathrm{H}, \mathrm{H}_{\mathrm{ar}}\right), 7.02$ (dd, $J=2.0$, $8.5 \mathrm{~Hz}, 1 \mathrm{H}, \mathrm{H}_{\text {ar. }}$ ), 7.09 (d, $J=7.5 \mathrm{~Hz}, 1 \mathrm{H}, \mathrm{H}_{\text {ar. }}$ ), 7.28 (d, $J=$ $7.5 \mathrm{~Hz}, 1 \mathrm{H}, \mathrm{H}_{\mathrm{ar}}$ ), 7.51 (d, $J=8.5 \mathrm{~Hz}, 1 \mathrm{H}, \mathrm{H}_{\mathrm{ar}}$ ), $7.63(\mathrm{~s}, 1 \mathrm{H}$, $\left.\mathrm{H}_{\mathrm{ar}}\right), 7.70-7.74\left(\mathrm{~m}, 2 \mathrm{H}, \mathrm{H}_{\mathrm{ar}}\right), 10.69$ (s, 1H, NH), 11.81 (s, $1 \mathrm{H}, \mathrm{NH}), 11.95$ (s, 1H, NH); ${ }^{13} \mathrm{C}$ NMR (DMSO- $\left.d_{6}\right) \mathrm{ppm}$ : $56.3\left(\mathrm{OCH}_{3}\right), 102.6,111.6,112.6,112.9,113.1,116.4,118.7$, $120.7,122.8,125.1,127.5,137.5,138.1,154.9,155.9\left(\mathrm{C}_{\mathrm{ar}}\right.$, $\left.\mathrm{CH}_{\text {ar. }}, \mathrm{C}=\mathrm{N}\right), 163.8,165.4(2 \times \mathrm{C}=\mathrm{O}) ; \mathrm{MS} \mathrm{m} / \mathrm{z}: 333$ [M-H] $]^{-}$. $N^{\prime}$-[(3Z)-1-Methyl-2-oxo-1,2-dihydro-3H-indol-3-ylidene]-1H-indole-2-carbohydrazide (5f): Yellow powder, $\mathrm{m}$. p. $258-260{ }^{\circ} \mathrm{C}$ (yield $41 \%$ ); ${ }^{1} \mathrm{H}$ NMR (DMSO- $d_{6}$ ) ppm: 3.23 $\left(\mathrm{s}, 3 \mathrm{H}, \mathrm{NCH}_{3}\right), 7.13\left(\mathrm{~d}, J=8.0 \mathrm{~Hz}, 1 \mathrm{H}, \mathrm{H}_{\mathrm{ar}}\right), 7.17-7.21(\mathrm{~m}$, $\left.2 \mathrm{H}, \mathrm{H}_{\text {ar. }}\right), 7.28$ (d, $\left.J=7.5 \mathrm{~Hz}, 1 \mathrm{H}, \mathrm{H}_{\mathrm{ar}}\right), 7.47-7.53(\mathrm{~m}, 2 \mathrm{H}$, $\left.\mathrm{H}_{\mathrm{ar}}\right), 7.61\left(\mathrm{~s}, 1 \mathrm{H}, \mathrm{H}_{\mathrm{ar}}\right), 7.73$ (d, $\left.J=8.0 \mathrm{~Hz}, 1 \mathrm{H}, \mathrm{H}_{\mathrm{ar}}\right), 8.09$ (d, $J=7.5 \mathrm{~Hz}, 1 \mathrm{H}, \mathrm{H}_{\mathrm{ar}}$ ), 11.76 (s, 1H, NH), 11.98 (s, 1H, NH); ${ }^{13} \mathrm{C}$ NMR (DMSO- $\left.d_{6}\right)$ ppm: $26.6\left(\mathrm{NCH}_{3}\right), 109.8,110.5$, $113.1,121.0,122.8,125.1,125.4,126.8,127.4,129.3$, 132.0, 133.1, 137.6, 144.1, $145.4\left(\mathrm{C}_{\text {ar. }}, \mathrm{CH}_{\text {ar. }}, \mathrm{C}=\mathrm{N}\right), 161.7$, $163.9(2 \times \mathrm{C}=\mathrm{O}) ; \mathrm{MS} \mathrm{m} / \mathrm{z}: 317[\mathrm{M}-1]^{-}$.

$N^{\prime}$-[(3Z)-5-Bromo-1-methyl-2-oxo-1,2-dihydro$3 H$-indol-3-ylidene]-1 $H$-indole-2-carbohydrazide $\quad(\mathbf{5 g})$ : Yellow powder, m.p. 291-293 ${ }^{\circ} \mathrm{C}$ (yield 63\%); ${ }^{1} \mathrm{H}$ NMR (DMSO-d $)_{6}$ ppm: $3.21\left(\mathrm{~s}, 3 \mathrm{H}, \mathrm{NCH}_{3}\right), 7.08-$ $7.15\left(\mathrm{~m}, 2 \mathrm{H}, \mathrm{H}_{\mathrm{ar}}\right), 7.28\left(\mathrm{~d}, J=7.5 \mathrm{~Hz}, 1 \mathrm{H}, \mathrm{H}_{\text {ar. }}\right), 7.51$ (d, $\left.J=8.5 \mathrm{~Hz}, 1 \mathrm{H}, \mathrm{H}_{\mathrm{ar}}\right), 7.63-7.68\left(\mathrm{~m}, 2 \mathrm{H}, \mathrm{H}_{\mathrm{ar}}\right), 7.73$ (d, $\left.J=8.0 \mathrm{~Hz}, 1 \mathrm{H}, \mathrm{H}_{\mathrm{ar}}\right), 8.38\left(\mathrm{~s}, 1 \mathrm{H}, \mathrm{H}_{\mathrm{ar}}\right), 11.93$ (s, $1 \mathrm{H}, \mathrm{NH}), 11.95(\mathrm{~s}, 1 \mathrm{H}, \mathrm{NH}) ;{ }^{13} \mathrm{C} \mathrm{NMR}\left(\mathrm{DMSO}-d_{6}\right)$ ppm: $26.6\left(\mathrm{NCH}_{3}\right), 111.6,112.9,114.5,120.6,122.8$, $123.3,125.2,127.5,128.9,134.1,135.0,137.5,138.1$, $143.1,144.5\left(\mathrm{C}_{\text {ar. }}, \mathrm{CH}_{\text {ar., }} \mathrm{C}=\mathrm{N}\right), 161.4,162.1(2 \times$ $\mathrm{C}=\mathrm{O}) ; \mathrm{MS} \mathrm{m} / \mathrm{z}: 395[\mathrm{M}-\mathrm{H}]^{-}, 397[\mathrm{M}-\mathrm{H}+2]^{-}$.

$N^{\prime}$-[(3Z)-5-Chloro-1-methyl-2-oxo-1,2-dihydro$3 H$-indol-3-ylidene]-1H-indole-2-carbohydrazide $\mathbf{( 5 h ) : ~}$ Yellow powder, m.p. 292-294 ${ }^{\circ} \mathrm{C}$ (yield 82\%); ${ }^{1} \mathrm{H}$ NMR (DMSO-d $d_{6}$ ppm: $3.22\left(\mathrm{~s}, 3 \mathrm{H}, \mathrm{NCH}_{3}\right), 7.10$ (d, $\left.J=8.0,1 \mathrm{H}, \mathrm{H}_{\mathrm{ar}}\right), 7.13\left(\mathrm{~d}, J=4.0 \mathrm{~Hz}, 1 \mathrm{H}, \mathrm{H}_{\mathrm{ar} .}\right)$, 7.27-7.31 (m,1H, $\left.\mathrm{H}_{\text {ar. }}\right), 7.51\left(\mathrm{~d}, J=8.5 \mathrm{~Hz}, 1 \mathrm{H}, \mathrm{H}_{\mathrm{ar} .}\right)$, 7.54 (dd, $\left.J=8.5,2.0 \mathrm{~Hz}, 1 \mathrm{H}, \mathrm{H}_{\mathrm{ar}}\right), 7.66$ (s, $\left.1 \mathrm{H}, \mathrm{H}_{\mathrm{ar}}\right)$, $7.73\left(\mathrm{~d}, J=8.0,1 \mathrm{H}, \mathrm{H}_{\mathrm{ar}}\right), 8.26\left(\mathrm{~s}, 1 \mathrm{H}, \mathrm{H}_{\mathrm{ar}}\right), 11.92(\mathrm{~s}$, $1 \mathrm{H}, \mathrm{NH}), 11.95(\mathrm{~s}, 1 \mathrm{H}, \mathrm{NH}) ;{ }^{13} \mathrm{C} \mathrm{NMR}\left(\mathrm{DMSO}-d_{6}\right)$ 
ppm: $26.6\left(\mathrm{NCH}_{3}\right), 109.3,111.1,112.0,112.9,120.7$, $122.8,125.2,126.3,126.8,127.5,127.9,132.2,137.6$, $142.7,144.1\left(\mathrm{C}_{\text {ar. }}, \mathrm{CH}_{\text {ar., }} \mathrm{C}=\mathrm{N}\right), 159.4,161.5(2 \times$ $\mathrm{C}=\mathrm{O}) ; \mathrm{MS} \mathrm{m} / \mathrm{z}: 351[\mathrm{M}-\mathrm{H}]^{-}$.

$N^{\prime}$-[(3Z)-5-Fluoro-1-methyl-2-oxo-1,2-dihydro-3Hindol-3-ylidene]-1 $H$-indole-2-carbohydrazide (5i): Yellow powder, m.p. 283-285 ${ }^{\circ} \mathrm{C}$ (yield 54\%); ${ }^{1} \mathrm{H} \quad \mathrm{NMR}$ (DMSO-d $)_{6}$ ppm: $3.22\left(\mathrm{~s}, 3 \mathrm{H}, \mathrm{NCH}_{3}\right), 7.09-7.14(\mathrm{~m}$, $2 \mathrm{H}, \mathrm{H}_{\mathrm{ar}}$ ), 7.27-7.30 (m, $\left.1 \mathrm{H}, \mathrm{H}_{\mathrm{ar}}\right), 7.37$ (ddd, $J=2.5$, 9.0, 9.0, 1H, $\mathrm{H}_{\mathrm{ar}}$ ), $7.51\left(\mathrm{~d}, J=8.0 \mathrm{~Hz}, 1 \mathrm{H}, \mathrm{H}_{\mathrm{ar}}\right), 7.67$ (s, $\left.1 \mathrm{H}, \mathrm{H}_{\mathrm{ar}}\right), 7.74$ (d, $\left.J=8.0 \mathrm{~Hz}, 1 \mathrm{H}, \mathrm{H}_{\mathrm{ar}}\right), 8.07$ (d, $J=7.5$ $\mathrm{Hz}, 1 \mathrm{H}, \mathrm{H}_{\mathrm{ar}}$ ), 11.86 (s, 1H, NH), 11.96 (s, 1H, NH); ${ }^{13} \mathrm{C}$ NMR (DMSO- $\left.d_{6}\right)$ ppm: $26.7\left(\mathrm{NCH}_{3}\right), 109.4,110.6$, $113.1,114.2,115.7,118.9,120.7,122.8,125.2,125.4$, 127.5, 129.0, 137.6, $141.8\left(\mathrm{C}_{\text {ar. }}, \mathrm{CH}_{\text {ar., }} \mathrm{C}=\mathrm{N}\right), 158.3$ $\left(\mathrm{C}_{1^{\prime}-\mathrm{F}}, J=235.6 \mathrm{~Hz}, \mathrm{C}_{\mathrm{ar}}\right), 161.8,163.9(2 \times \mathrm{C}=\mathrm{O})$; MS m/z: $335[\mathrm{M}-\mathrm{H}]^{-}$.

$N^{\prime}$-[(3Z)-5-Methoxy-1-methyl-2-oxo-1,2-dihydro-3Hindol-3-ylidene]-1 $H$-indole-2-carbohydrazide (5j): Orange powder, m.p. 279-281 ${ }^{\circ} \mathrm{C}$ (yield 86\%); ${ }^{1} \mathrm{H} \quad \mathrm{NMR}$ (DMSO-d $)_{6}$ ppm: $3.19\left(\mathrm{~s}, 3 \mathrm{H}, \mathrm{NCH}_{3}\right), 3.84(\mathrm{~s}, 3 \mathrm{H}$, $\left.\mathrm{OCH}_{3}\right), 7.04\left(\mathrm{~d}, J=8.5 \mathrm{~Hz}, 1 \mathrm{H}, \mathrm{H}_{\mathrm{ar}}\right), 7.08-7.12(\mathrm{~m}, 2 \mathrm{H}$, $\left.\mathrm{H}_{\mathrm{ar}}\right)$, 7.27-7.30 (m, 1H, $\left.\mathrm{H}_{\mathrm{ar}}\right), 7.63\left(\mathrm{~s}, 1 \mathrm{H}, \mathrm{H}_{\mathrm{ar}}\right), 7.66(\mathrm{~d}$, $\left.J=8.0 \mathrm{~Hz}, 1 \mathrm{H}, \mathrm{H}_{\mathrm{ar}}\right), 7.72-7.77\left(\mathrm{~m}, 2 \mathrm{H}, \mathrm{H}_{\mathrm{ar}}\right), 11.86(\mathrm{~s}$, $1 \mathrm{H}, \mathrm{NH}), 11.96$ (s, 1H, NH); ${ }^{13} \mathrm{C}$ NMR (DMSO-d $)$ ppm: $26.6\left(\mathrm{NCH}_{3}\right), 56.4\left(\mathrm{OCH}_{3}\right), 110.2,111.3,112.9,113.4$, $115.8,117.9,120.7,122.8,125.1,127.5,129.2,137.5$, 139.2, $155.5\left(\mathrm{C}_{\text {ar. }}, \mathrm{CH}_{\text {ar., }} \mathrm{C}=\mathrm{N}\right), 161.7,163.9(2 \times \mathrm{C}=\mathrm{O})$; $\mathrm{MS} \mathrm{m} / \mathrm{z}: 347[\mathrm{M}-\mathrm{H}]^{-}$.

$N^{\prime}$-[(3Z)-1-Benzyl-2-oxo-1,2-dihydro-3H-indol-3-ylidene]-1 $H$-indole-2-carbohydrazide (5k): Yellow powder, $\mathrm{m}$. p. $249-251{ }^{\circ} \mathrm{C}$ (yield $56 \%$ ); ${ }^{1} \mathrm{H}$ NMR (DMSO- $d_{6}$ ) ppm: 5.02 $\left(\mathrm{s}, 2 \mathrm{H}, \mathrm{CH}_{2}\right), 7.06\left(\mathrm{~d}, J=8.0 \mathrm{~Hz}, 1 \mathrm{H}, \mathrm{H}_{\mathrm{ar}}\right), 7.10-7.13(\mathrm{~m}, 1 \mathrm{H}$, $\left.\mathrm{H}_{\mathrm{ar} .}\right)$, 7.16-7.21 (m, 1H, $\left.\mathrm{H}_{\mathrm{ar} .}\right), 7.28-7.32\left(\mathrm{~m}, 2 \mathrm{H}, \mathrm{H}_{\mathrm{ar} .}\right)$, 7.35-7.38 (m, 3H, $\left.\mathrm{H}_{\mathrm{ar}}\right), 7.42-7.45\left(\mathrm{~m}, 2 \mathrm{H}, \mathrm{H}_{\mathrm{ar}}\right), 7.51(\mathrm{~d}$, $\left.J=8.5 \mathrm{~Hz}, 1 \mathrm{H}, \mathrm{H}_{\mathrm{ar}}\right), 7.65\left(\mathrm{~s}, 1 \mathrm{H}, \mathrm{H}_{\mathrm{ar}}\right), 7.74(\mathrm{~d}, J=7.5 \mathrm{~Hz}$, $\left.1 \mathrm{H}, \mathrm{H}_{\mathrm{ar}}\right), 8.14$ (d, J=7.5 Hz, $\left.1 \mathrm{H}, \mathrm{H}_{\mathrm{ar}}\right), 11.80$ (s, 1H, NH), $12.00(\mathrm{~s}, 1 \mathrm{H}, \mathrm{NH}) ;{ }^{13} \mathrm{C}$ NMR (DMSO- $\left.d_{6}\right) \mathrm{ppm}: 43.2\left(\mathrm{CH}_{2}\right)$, 110.4, 113.0, 115.7, 120.7, 120.9, 121.3, 122.7, 123.0, 127.5, 127.7, 127.9, 128.0, 129.2, 134.1, 136.7, 137.7, 144.3, 146.8, $147.5\left(\mathrm{C}_{\text {ar. }}, \mathrm{CH}_{\mathrm{ar}}, \mathrm{C}=\mathrm{N}\right), 162.4,164.2(2 \times \mathrm{C}=\mathrm{O}) ; \mathrm{MS} \mathrm{m} / \mathrm{z}$ : $393[\mathrm{M}-\mathrm{H}]^{-}$.

$N^{\prime}$-[(3Z)-1-Benzyl-5-bromo-2-oxo-1,2-dihydro-3Hindol-3-ylidene]-1H-indole-2-carbohydrazide (51): Yellow powder, m.p. 268-270 ${ }^{\circ} \mathrm{C}$ (yield 51\%); ${ }^{1} \mathrm{H} \quad \mathrm{NMR}$ (DMSO-d $d_{6}$ ppm: $5.02\left(\mathrm{~s}, 2 \mathrm{H}, \mathrm{CH}_{2}\right), 7.01(\mathrm{~d}, J=8.5 \mathrm{~Hz}, 1 \mathrm{H}$, $\left.\mathrm{H}_{\mathrm{ar}}\right), 7.10\left(\mathrm{~d}, J=7.5 \mathrm{~Hz}, 1 \mathrm{H}, \mathrm{H}_{\mathrm{ar}}\right), 7.29$ (d, $J=7.5 \mathrm{~Hz}, 2 \mathrm{H}$, $\left.\mathrm{H}_{\mathrm{ar} .}\right), 7.36-7.38\left(\mathrm{~m}, 3 \mathrm{H}, \mathrm{H}_{\mathrm{ar}}\right), 7.42\left(\mathrm{~d}, J=7.5 \mathrm{~Hz}, 1 \mathrm{H}, \mathrm{H}_{\mathrm{ar} .}\right)$, $7.52\left(\mathrm{~d}, J=8.0 \mathrm{~Hz}, 1 \mathrm{H}, \mathrm{H}_{\mathrm{ar}}\right), 7.59\left(\mathrm{~s}, 1 \mathrm{H}, \mathrm{H}_{\mathrm{ar}}\right), 7.71-7.77(\mathrm{~m}$, $2 \mathrm{H}, \mathrm{H}_{\mathrm{ar}}$ ), 8.44 (s $1 \mathrm{H}, \mathrm{H}_{\mathrm{ar}}$ ), 11.99 (s, 1H, NH), 12.20 (s, 1H, $\mathrm{NH}) ;{ }^{13} \mathrm{C}$ NMR (DMSO-d $)$ ppm: $43.3\left(\mathrm{CH}_{2}\right), 110.3,112.1$, 113.1, 114.8, 120.7, 127.7, 127.9, 128.2, 129.2, 134.1, 135.8, $136.4,136.6,137.6,138.1,142.1,143.3,148.7,156.5\left(\mathrm{C}_{\mathrm{ar}}\right.$, $\left.\mathrm{CH}_{\text {ar. }}, \mathrm{C}=\mathrm{N}\right), 161.5,162.6(2 \times \mathrm{C}=\mathrm{O}) ; \mathrm{MS} \mathrm{m} / \mathrm{z}: 471[\mathrm{M}-\mathrm{H}]^{-}$; $473[\mathrm{M}-\mathrm{H}+2]^{-}$.

$N^{\prime}$-[(3Z)-1-Benzyl-5-chloro-2-oxo-1,2-dihydro-

3H-indol-3-ylidene]-1 $H$-indole-2-carbohydrazide $\quad(\mathbf{5 m})$ : Yellow powder, m.p. 268-270 ${ }^{\circ} \mathrm{C}$ (yield 54\%); ${ }^{1} \mathrm{H}$ NMR (DMSO-d $)_{6}$ ppm: $5.02\left(\mathrm{~s}, 2 \mathrm{H}, \mathrm{CH}_{2}\right), 7.06(\mathrm{~d}, J=8.5 \mathrm{~Hz}$, $\left.1 \mathrm{H}, \mathrm{H}_{\mathrm{ar}}\right), 7.11-7.13\left(\mathrm{~m}, 1 \mathrm{H}, \mathrm{H}_{\mathrm{ar}}\right), 7.28-7.31\left(\mathrm{~m}, 2 \mathrm{H}, \mathrm{H}_{\mathrm{ar} .}\right)$, $7.35-7.37$ (m, 3H, $\left.\mathrm{H}_{\text {ar. }}\right), 7.43\left(\mathrm{~d}, J=7.0 \mathrm{~Hz}, 1 \mathrm{H}, \mathrm{H}_{\mathrm{ar}}\right)$, 7.46-7.47 (m, 1H, $\left.\mathrm{H}_{\text {ar. }}\right), 7.52\left(\mathrm{~d}, J=8.0 \mathrm{~Hz}, 1 \mathrm{H}, \mathrm{H}_{\mathrm{ar} .}\right), 7.71$ $\left(\mathrm{s}, 1 \mathrm{H}, \mathrm{H}_{\mathrm{ar}}\right), 7.74\left(\mathrm{~d}, J=8.0 \mathrm{~Hz}, 1 \mathrm{H}, \mathrm{H}_{\mathrm{ar}}\right), 8.32(\mathrm{~s}, 1 \mathrm{H}$, $\left.\mathrm{H}_{\mathrm{ar}}\right), 11.97(\mathrm{~s}, 1 \mathrm{H}, \mathrm{NH}), 12.18(\mathrm{~s}, 1 \mathrm{H}, \mathrm{NH}) ;{ }^{13} \mathrm{C} \mathrm{NMR}$ (DMSO-d $\left.{ }_{6}\right)$ ppm: $43.3\left(\mathrm{CH}_{2}\right), 111.7,112.5,112.9,113.1$, $120.7,121.6,127.5,127.7,127.9,128.0,128.2,129.2$, $135.9,136.4,137.6,138.1,141.7,144.4,149.6\left(C_{a r}\right.$, $\left.\mathrm{CH}_{\text {ar. }}, \mathrm{C}=\mathrm{N}\right), 159.7,161.6(2 \times \mathrm{C}=\mathrm{O}) ; \mathrm{MS} \mathrm{m} / \mathrm{z}: 427$ $[\mathrm{M}-\mathrm{H}]^{-} ; 429[\mathrm{M}-\mathrm{H}+2]^{-}$.

$N^{\prime}$-[(3Z)-1-Benzyl-5-fluoro-2-oxo-1,2-dihydro-3Hindol-3-ylidene]-1H-indole-2-carbohydrazide (5n): Yellow powder, m.p. 251-253 ${ }^{\circ} \mathrm{C}$ (yield 49\%); ${ }^{1} \mathrm{H} \quad \mathrm{NMR}$ (DMSO-d $)_{6}$ ppm: $5.02\left(\mathrm{~s}, 2 \mathrm{H}, \mathrm{CH}_{2}\right), 7.04-7.06(\mathrm{~m}, 1 \mathrm{H}$, $\mathrm{H}_{\mathrm{ar}}$ ), 7.09-7.13 (m, 1H, $\left.\mathrm{H}_{\mathrm{ar}}\right), 7.28-7.32\left(\mathrm{~m}, 3 \mathrm{H}, \mathrm{H}_{\mathrm{ar}}\right)$, 7.34-7.39 (m, 4H, $\mathrm{H}_{\text {ar. }}$ ), 7.29 (d, $J=8.5 \mathrm{~Hz}, 1 \mathrm{H}, \mathrm{H}_{\mathrm{ar}}$ ), $7.72\left(\mathrm{~s}, 1 \mathrm{H}, \mathrm{H}_{\mathrm{ar}}\right), 7.75\left(\mathrm{~d}, J=8.0 \mathrm{~Hz}, 1 \mathrm{H}, \mathrm{H}_{\mathrm{ar}}\right), 8.13(\mathrm{~d}$, $\left.J=7.5 \mathrm{~Hz}, 1 \mathrm{H}, \mathrm{H}_{\mathrm{ar}}\right), 11.91(\mathrm{~s}, 1 \mathrm{H}, \mathrm{NH}), 11.99$ (s, 1H, $\mathrm{NH}) ;{ }^{13} \mathrm{C}$ NMR (DMSO-d $)$ ppm: $43.3\left(\mathrm{CH}_{2}\right), 110.9$, $111.1,113.0,114.4,119.0,120.7,122.8,125.3,127.5$, $127.7,127.9,128.0,128.9,129.2,136.5,137.6,139.9$, $140.6\left(\mathrm{C}_{\mathrm{ar}}, \mathrm{CH}_{\mathrm{ar}}, \mathrm{C}=\mathrm{N}\right), 158.4\left(\mathrm{C}_{1^{\prime}-\mathrm{F}}, J=237.9 \mathrm{~Hz}\right.$, $\left.\mathrm{C}_{\mathrm{ar} .}\right), 161.9,164.2(2 \times \mathrm{C}=\mathrm{O}) ; \mathrm{MS} \mathrm{m} / \mathrm{z}: 411[\mathrm{M}-\mathrm{H}]^{-}$.

$N^{\prime}$-[(3Z)-1-Benzyl-5-methoxy-2-oxo-1,2-dihydro-

$3 H$-indol-3-ylidene]-1 $H$-indole-2-carbohydrazide $\quad$ (5o): Orange powder, m.p. 268-270 ${ }^{\circ} \mathrm{C}$ (yield 64\%); ${ }^{1} \mathrm{H}$ NMR (DMSO-d $)_{6}$ ppm: $3.81\left(\mathrm{~s}, 3 \mathrm{H}, \mathrm{OCH}_{3}\right), 4.99\left(\mathrm{~s}, 2 \mathrm{H}, \mathrm{CH}_{2}\right)$, $6.95\left(\mathrm{~d}, J=8.5 \mathrm{~Hz}, 1 \mathrm{H}, \mathrm{H}_{\mathrm{ar}}\right), 7.02(\mathrm{dd}, J=2.0,8.5 \mathrm{~Hz}$, $\left.1 \mathrm{H}, \mathrm{H}_{\mathrm{ar}}\right), 7.10$ (d, $\left.J=8.0 \mathrm{~Hz}, 1 \mathrm{H}, \mathrm{H}_{\mathrm{ar}}\right), 7.26-7.31(\mathrm{~m}, 2 \mathrm{H}$, $\left.\mathrm{H}_{\mathrm{ar}}\right), 7.34-7.38\left(\mathrm{~m}, 4 \mathrm{H}, \mathrm{H}_{\mathrm{ar}}\right), 7.52(\mathrm{~d}, J=8.0 \mathrm{~Hz}, 1 \mathrm{H}$, $\mathrm{H}_{\mathrm{ar}}$ ), 7.68 (s, 1H, $\mathrm{H}_{\mathrm{ar}}$ ), 7.74 (d, $\left.J=8.0 \mathrm{~Hz}, 1 \mathrm{H}, \mathrm{H}_{\mathrm{ar} .}\right), 7.78$ (s, 1H, $\left.\mathrm{H}_{\mathrm{ar}}\right), 11.92$ (s, 1H, NH), $\left.11.98 \mathrm{~s}, 1 \mathrm{H}, \mathrm{NH}\right)$; ${ }^{13} \mathrm{C}$ NMR (DMSO- $\left.d_{6}\right)$ ppm: $43.1\left(\mathrm{CH}_{2}\right), 56.4\left(\mathrm{OCH}_{3}\right)$, $110.9,113.0,116.1,120.7,122.8,125.2,127.6,127.7$, $127.9,128.0,129.2,129.3,136.7,136.8,137.7,138.0$, 138.1, 144.1, $155.6\left(\mathrm{C}_{\text {ar. }}, \mathrm{CH}_{\text {ar. }}, \mathrm{C}=\mathrm{N}\right), 156.5,164.1(2 \times$ $\mathrm{C}=\mathrm{O}) ; \mathrm{MS} \mathrm{m} / \mathrm{z}: 423[\mathrm{M}-\mathrm{H}]^{-}$. 
$N^{\prime}$-[(3Z)-1-(4-Chlorobenzyl)-2-oxo-1,2-dihydro-3Hindol-3-ylidene]-1 $H$-indole-2-carbohydrazide (5p): Yellow powder, m.p. 261-263 ${ }^{\circ} \mathrm{C}$ (yield 65\%); ${ }^{1} \mathrm{H}$ NMR (DMSO- $d_{6}$ ) ppm: $5.02\left(\mathrm{~s}, 2 \mathrm{H}, \mathrm{CH}_{2}\right), 7.06(\mathrm{~d}, J=8.0 \mathrm{~Hz}$, $\left.1 \mathrm{H}, \mathrm{H}_{\mathrm{ar}}\right), 7.10-7.14\left(\mathrm{~m}, 1 \mathrm{H}, \mathrm{H}_{\mathrm{ar}}\right), 7.19-7.21\left(\mathrm{~m}, 1 \mathrm{H}, \mathrm{H}_{\mathrm{ar}}\right)$, 7.28-7.32 (m, 1H, $\left.\mathrm{H}_{\text {ar. }}\right), 7.40-7.44\left(\mathrm{~m}, 3 \mathrm{H}, \mathrm{H}_{\text {ar. }}\right), 7.51(\mathrm{~d}$, $\left.J=8.0 \mathrm{~Hz}, 2 \mathrm{H}, \mathrm{H}_{\text {ar. }}\right), 7.65\left(\mathrm{~s}, 1 \mathrm{H}, \mathrm{H}_{\text {ar }}\right), 7.72-7.75(\mathrm{~m}, 1 \mathrm{H}$, $\left.\mathrm{H}_{\text {ar. }}\right), 7.77\left(\mathrm{~d}, J=8.0 \mathrm{~Hz}, 1 \mathrm{H}, \mathrm{H}_{\text {ar. }}\right), 8.15(\mathrm{~d}, J=7.5 \mathrm{~Hz}$, $\left.1 \mathrm{H}, \mathrm{H}_{\mathrm{ar}}\right), 11.81(\mathrm{~s}, 1 \mathrm{H}, \mathrm{NH}), 11.99(\mathrm{~s}, 1 \mathrm{H}, \mathrm{NH}) ;{ }^{13} \mathrm{C} \mathrm{NMR}$ (DMSO- $\left.d_{6}\right)$ ppm: $42.6\left(\mathrm{CH}_{2}\right), 110.9,113.0,119.9,123.1$, $125.2,125.4,127.5,129.2,129.7,129.9,131.9,132.6$, $132.8,135.2,135.7,137.7,138.0,142.9,144.1\left(\mathrm{C}_{\mathrm{ar}}\right.$, $\left.\mathrm{CH}_{\text {ar. }}, \mathrm{C}=\mathrm{N}\right), 161.8,164.2(2 \times \mathrm{C}=\mathrm{O}) ; \mathrm{MS} \mathrm{m} / \mathrm{z}: 427$ $[\mathrm{M}-\mathrm{H}]^{-} ; 429[\mathrm{M}-\mathrm{H}+2]^{-}$.

$N^{\prime}$-[(3Z)-1-(4-Cyanobenzyl)-2-oxo-1,2-dihydro-3Hindol-3-ylidene]- $1 H$-indole-2-carbohydrazide (5q): Yellow powder, m.p. 266-268 ${ }^{\circ} \mathrm{C}$ (yield 57\%); ${ }^{1} \mathrm{H} \quad \mathrm{NMR}$ (DMSO- $d_{6}$ ) ppm: 5.13 (s, $\left.2 \mathrm{H}, \mathrm{CH}_{2}\right), 7.05(\mathrm{~d}, J=7.5 \mathrm{~Hz}$, $1 \mathrm{H}, \mathrm{H}_{\text {ar. }}$ ), 7.10-7.13 (m, 1H, $\mathrm{H}_{\text {ar. }}$ ), 7.20 (ddd, $J=2.0,7.5$, $\left.7.5 \mathrm{~Hz}, 1 \mathrm{H}, \mathrm{H}_{\text {ar. }}\right), 7.39-7.43\left(\mathrm{~m}, 1 \mathrm{H}, \mathrm{H}_{\text {ar. }}\right), 7.56(\mathrm{~d}, J=8.5$ $\left.\mathrm{Hz}, 2 \mathrm{H}, \mathrm{H}_{\mathrm{ar}}\right), 7.63-7.65\left(\mathrm{~m}, 3 \mathrm{H}, \mathrm{H}_{\text {ar }}\right), 7.77$ (d, $J=8.0 \mathrm{~Hz}$, $\left.1 \mathrm{H}, \mathrm{H}_{\mathrm{ar} .}\right), 7.84\left(\mathrm{~d}, J=3.0 \mathrm{~Hz}, 1 \mathrm{H}, \mathrm{H}_{\mathrm{ar}}\right), 7.85(\mathrm{~d}, J=3.0$ $\left.\mathrm{Hz}, 1 \mathrm{H}, \mathrm{H}_{\mathrm{ar}}\right), 8.17$ (d, $\left.J=7.5 \mathrm{~Hz}, 1 \mathrm{H}, \mathrm{H}_{\mathrm{ar}}\right), 11.82(\mathrm{~s}, 1 \mathrm{H}$, $\mathrm{NH}), 11.99(\mathrm{~s}, 1 \mathrm{H}, \mathrm{NH}) ;{ }^{13} \mathrm{C}$ NMR (DMSO- $\left.d_{6}\right) \mathrm{ppm}: 42.9$ $\left(\mathrm{CH}_{2}\right), 110.8,110.9,113.0,115.8,119.9,120.7,122.7$, $123.2,125.4,127.1,127.5,128.6,128.8,133.1,133.2$, 137.7, 138.0, 141.9, 142.5, $142.8\left(\mathrm{C}_{\text {ar. }}, \mathrm{CH}_{\text {ar. }}, 2 \times \mathrm{CN}\right)$, 161.9, $164.2(2 \times \mathrm{C}=\mathrm{O}) ; \mathrm{MS} \mathrm{m} / \mathrm{z}: 418[\mathrm{M}-\mathrm{H}]^{-}$.

$N^{\prime}$-[(3Z)-1-(4-Methylbenzyl)-2-oxo-1,2-dihydro-3Hindol-3-ylidene]-1 $H$-indole-2-carbohydrazide (5r): Yellow powder, m.p. 256-258 ${ }^{\circ} \mathrm{C}$ (yield 40\%); ${ }^{1} \mathrm{H} \quad \mathrm{NMR}$ (DMSO- $d_{6}$ ) ppm: $2.27\left(\mathrm{~s}, 3 \mathrm{H}, \mathrm{CH}_{3}\right), 4.97\left(\mathrm{~s}, 2 \mathrm{H}, \mathrm{CH}_{2}\right)$, $7.04\left(\mathrm{~d}, J=8.0 \mathrm{~Hz}, 1 \mathrm{H}, \mathrm{H}_{\text {ar. }}\right), 7.10-7.13\left(\mathrm{~m}, 1 \mathrm{H}, \mathrm{H}_{\mathrm{ar}}\right), 7.16$ $\left(\mathrm{d}, J=8.5 \mathrm{~Hz}, 2 \mathrm{H}, \mathrm{H}_{\mathrm{ar}}\right), 7.27\left(\mathrm{~d}, J=8.5 \mathrm{~Hz}, 2 \mathrm{H}, \mathrm{H}_{\text {ar. }}\right)$, 7.29-7.34 (m, 1H, $\left.\mathrm{H}_{\text {ar. }}\right), 7.41-7.44\left(\mathrm{~m}, 1 \mathrm{H}, \mathrm{H}_{\mathrm{ar}}\right), 7.52(\mathrm{~d}$, $\left.J=8.5 \mathrm{~Hz}, 1 \mathrm{H}, \mathrm{H}_{\text {ar. }}\right), 7.65\left(\mathrm{~s}, 1 \mathrm{H}, \mathrm{H}_{\text {ar. }}\right), 7.74(\mathrm{~d}, J=8.0 \mathrm{~Hz}$, $\left.1 \mathrm{H}, \mathrm{H}_{\text {ar }}\right), 8.13\left(\mathrm{~d}, J=7.5 \mathrm{~Hz}, 1 \mathrm{H}, \mathrm{H}_{\text {ar. }}\right), 11.80(\mathrm{~s}, 1 \mathrm{H}, \mathrm{NH})$, $12.00(\mathrm{~s}, 1 \mathrm{H}, \mathrm{NH}) ;{ }^{13} \mathrm{C}$ NMR (DMSO- $\left.d_{6}\right)$ ppm: 21.1 $\left(\mathrm{CH}_{3}\right), 43.0\left(\mathrm{CH}_{2}\right), 110.4,113.0,115.7,120.7,122.7$, $123.0,125.2,127.0,127.5,127.7,127.9,129.1,129.2$, $129.8,132.9,133.6,137.2,137.7,144.3\left(\mathrm{C}_{\text {ar. }}, \mathrm{CH}_{\text {ar. }}\right.$, $\mathrm{C}=\mathrm{N}), 162.5,164.1(2 \times \mathrm{C}=\mathrm{O}) ; \mathrm{MS} \mathrm{m} / \mathrm{z}: 407[\mathrm{M}-\mathrm{H}]^{-}$.

$N^{\prime}$-[(3Z)-2-Oxo-1-phenyl-1,2-dihydro-3H-indol-3-ylidene]-1H-indole-2-carbohydrazide (5s): Yellow powder, $\mathrm{m}$. p. $277-279{ }^{\circ} \mathrm{C}$ (yield $55 \%$ ); ${ }^{1} \mathrm{H}$ NMR (DMSO- $d_{6}$ ) ppm: 6.84 (d, $\left.J=8.0 \mathrm{~Hz}, 1 \mathrm{H}, \mathrm{H}_{\mathrm{ar}}\right), 7.11-7.14$ (m, $\left.1 \mathrm{H}, \mathrm{H}_{\mathrm{ar}}\right), 7.24-7.31$ (m, 2H, $\mathrm{H}_{\text {ar. }}$ ), 7.44-7.47 (m, 1H, $\left.\mathrm{H}_{\text {ar. }}\right), 7.50-7.54(\mathrm{~m}, 4 \mathrm{H}$, $\left.\mathrm{H}_{\mathrm{ar} .}\right), 7.61-7.64\left(\mathrm{~m}, 2 \mathrm{H}, \mathrm{H}_{\mathrm{ar}}\right), 7.58\left(\mathrm{~s}, 1 \mathrm{H}, \mathrm{H}_{\mathrm{ar}}\right), 7.74(\mathrm{~d}$,
$\left.J=8.0 \mathrm{~Hz}, 1 \mathrm{H}, \mathrm{H}_{\text {ar. }}\right), 8.23\left(\mathrm{~d}, J=7.5 \mathrm{~Hz}, 1 \mathrm{H}, \mathrm{H}_{\text {ar }}\right), 11.89(\mathrm{~s}$, $1 \mathrm{H}, \mathrm{NH}), 12.01(\mathrm{~s}, 1 \mathrm{H}, \mathrm{NH}) ;{ }^{13} \mathrm{C}$ NMR (DMSO- $d_{6}$ ) ppm: $110.1,113.0,115.7,120.7,122.8,123.5,125.2,127.2$, 127.4, 127.5, 128.9, 130.2, 133.1, 134.2, 135.5, 137.3, 137.7, 141.8, $145.1\left(\mathrm{C}_{\text {ar. }}, \mathrm{CH}_{\text {ar. }}, \mathrm{C}=\mathrm{N}\right), 162.9,163.4(2 \times \mathrm{C}=\mathrm{O}) ; \mathrm{MS} \mathrm{m} / \mathrm{z}$ : $379[\mathrm{M}-\mathrm{H}]^{-}$.

\section{Pharmacological Evaluation}

Pharmacological assessment of the title compounds $\mathbf{5 a}-\mathbf{s}$ was carried out using previously reported protocols $\mathrm{s}^{39,40}$ and they are provided as Supporting materials. The human cancer cell lines were procured commercially from the American Type Culture Collection.

\section{Western Blot Analysis Materials}

Immunoblotting detection was performed with antibodies from Abcam against cyclin E1(clone number HE12, catalog number ab3927), cyclin D1 (clone number EP272Y, catalog number ab40754), cyclin B1 (clone number Y106, catalog number ab32053), $\beta$ actin (clone number mAbcam8226, catalog number ab8226), BTG1 (clone number EPR8274(2), catalog number ab151740), BAX (clone number 2D2, catalog numberab77566), bcl-2 (clone number E17, catalog number ab32124), and caspase-3 (catalog number ab13847).

\section{Methods}

A-549 non-small cell lung cancer (NSCLC) cells were plated in $25-\mathrm{cm}^{2}$ culture dishes at a seeding density of 5 $\times 10^{4} /$ dish before treatment with compound $\mathbf{5 m}$ for 24,48 , and $72 \mathrm{~h}$. Whole cell lysates were prepared using radioimmunoprecipitation assay buffer (Sigma-Aldrich, MO, USA). Protein concentrations were determined using the DC Protein Assay Kit (Bio-Rad, CA, USA). Twenty micrograms of treated and control lysates were loaded in each well of 4-20\% Mini-Protean TGX Gels (Bio-Rad) for electrophoresis and then transferred to a polyvinylidene difluoride membrane (Bio-Rad). The membrane was blocked with $5 \%(\mathrm{w} / \mathrm{v})$ skimmed milk prepared in Trisbuffered saline and Tween 20, incubated with primary antibodies overnight at $4{ }^{\circ} \mathrm{C}$, washed, and then incubated with a secondary, horseradish peroxidase-coupled antimouse or anti-rabbit antibody. Bands were visualized with a Western Bright ECL Kit for $5000 \mathrm{~cm}^{2}$ Membrane and Blue Basic Autoradiography Film (BioExpress, CA, USA). 


\section{Results and Discussion Chemistry}

The chemical structures of the title compounds $\mathbf{5} \mathbf{a}-\mathbf{s}$ and their intermediates are shown in Scheme 1. The free carboxylic group of indole-2-carboxylic acid (1) was esterified, which resulted in the corresponding methyl ester 2. ${ }^{41}$ Subsequently, compound $\mathbf{2}$ was subjected to hydrazinolysis with hydrazine hydrate, thereby forming the acid hydrazide 3 . $^{42}$ The target indole-isatin conjugates $\mathbf{5 a}-\mathbf{s}$ were respectively prepared by reacting the acid hydrazide $\mathbf{3}$ with the appropriate isatin derivatives $\mathbf{4 a}-\mathbf{n}$ in ethanol-containing drops of acetic acid. The stereochemistry of the title conjugates $\mathbf{5 a}-\mathbf{s}$ was assigned the $(Z)$-configuration, based on the previously documented X-ray results of structurally related analogous compounds. ${ }^{43,44}$

\section{Pharmacological Investigations}

\section{Antiproliferative Activity}

The cell growth-inhibitory activities of nineteen conjugates 5a-s were evaluated with human breast (ZR-75), colon (HT-29) and lung (A-549) tumor cells. Each test compound was assessed at $30 \mu \mathrm{M}$ in quadruplicate (Table 1). The title compounds $\mathbf{5 a}-\mathbf{s}$ displayed average antiproliferative activities against the ZR-75, HT-29, and A-549 cell lines of 20.3 to $95.9 \%$.

Table 2 shows the $\mathrm{IC}_{50}$ (inhibitory concentration $50 \%$ ) values determined for compounds $\mathbf{5 a - s}$ against the tested human cancer cell lines. Compound $\mathbf{5 m}$ had an average $\mathrm{IC}_{50}$ value of $1.17 \mu \mathrm{M}$ against the tested human cancer cell lines, making it the most active candidate, with a potency approximately seven-fold greater than that of sunitinib (average $\mathrm{IC}_{50}$ value of

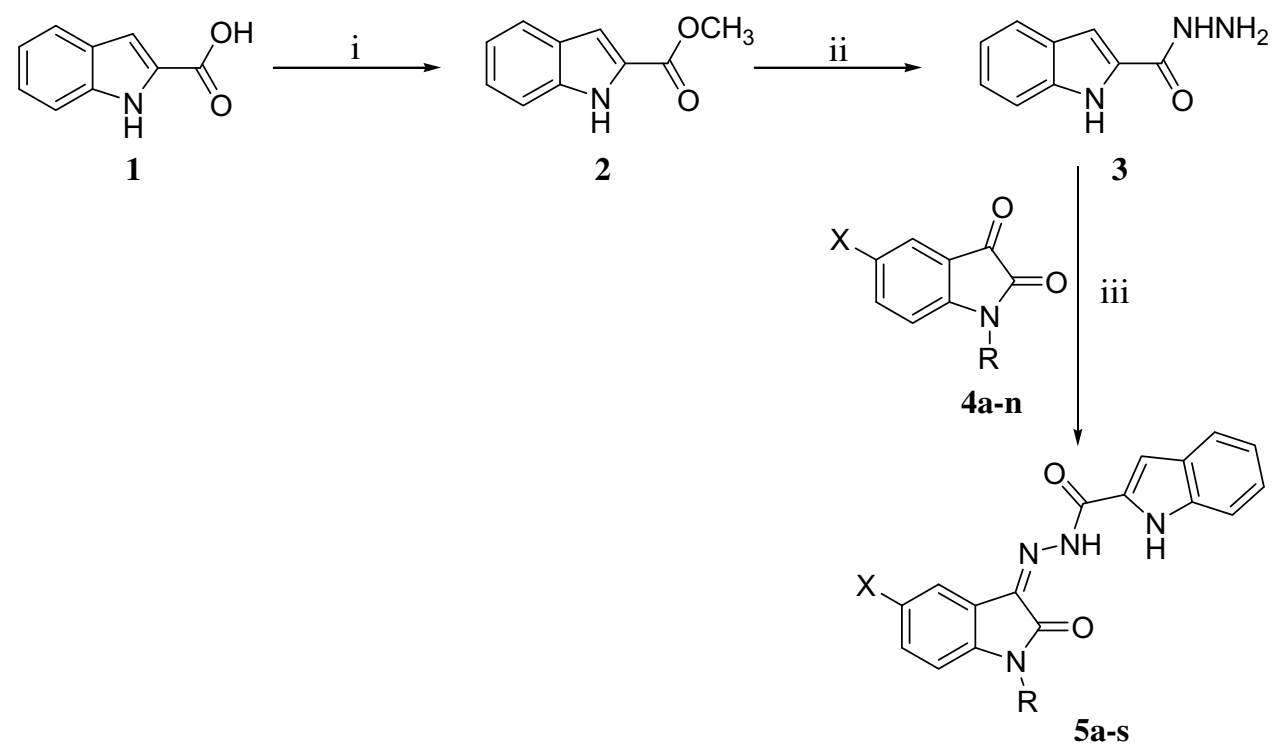

\begin{tabular}{|c|c|c|c|c|c|}
\hline $\begin{array}{c}\text { Compound } \\
\text { No. }\end{array}$ & $\mathrm{X}$ & $\mathrm{R}$ & $\begin{array}{c}\text { Compound } \\
\text { No. }\end{array}$ & $\mathrm{X}$ & $\mathrm{R}$ \\
\hline $\mathbf{5 a}$ & $\mathrm{H}$ & $\mathrm{H}$ & $\mathbf{5 k}$ & $\mathrm{H}$ & $\mathrm{C}_{6} \mathrm{H}_{5}-\mathrm{CH}_{2}$ \\
\hline $\mathbf{5 b}$ & $\mathrm{Br}$ & $\mathrm{H}$ & $\mathbf{5 l}$ & $\mathrm{Br}$ & $\mathrm{C}_{6} \mathrm{H}_{5}-\mathrm{CH}_{2}$ \\
\hline $\mathbf{5 c}$ & $\mathrm{Cl}$ & $\mathrm{H}$ & $\mathbf{5 m}$ & $\mathrm{Cl}$ & $\mathrm{C}_{6} \mathrm{H}_{5}-\mathrm{CH}_{2}$ \\
\hline $\mathbf{5 d}$ & $\mathrm{F}$ & $\mathrm{H}$ & $\mathbf{5 n}$ & $\mathrm{F}$ & $\mathrm{C}_{6} \mathrm{H}_{5}-\mathrm{CH}_{2}$ \\
\hline $\mathbf{5 e}$ & $\mathrm{OCH}_{3}$ & $\mathrm{H}$ & $\mathbf{5 o}$ & $\mathrm{OCH}_{3}$ & $\mathrm{C}_{6} \mathrm{H}_{5}-\mathrm{CH}_{2}$ \\
\hline $\mathbf{5 f}$ & $\mathrm{H}$ & $\mathrm{CH}_{3}$ & $\mathbf{5 p}$ & $\mathrm{H}$ & $4-\mathrm{Cl}_{6}-\mathrm{C}_{6} \mathrm{H}_{4}-\mathrm{CH}_{2}$ \\
\hline $\mathbf{5 g}$ & $\mathrm{Br}$ & $\mathrm{CH}_{3}$ & $\mathbf{5 q}$ & $\mathrm{H}$ & $4-\mathrm{CN}_{-} \mathrm{C}_{6} \mathrm{H}_{4}-\mathrm{CH}_{2}$ \\
\hline $\mathbf{5 h}$ & $\mathrm{Cl}$ & $\mathrm{CH}_{3}$ & $\mathbf{5 r}$ & $\mathrm{H}$ & $4-\mathrm{CH}_{3}-\mathrm{C}_{6} \mathrm{H}_{4}-\mathrm{CH}_{2}$ \\
\hline $\mathbf{5 i}$ & $\mathrm{F}$ & $\mathrm{CH}_{3}$ & $\mathbf{5 s}$ & $\mathrm{H}$ & $\mathrm{C}_{6} \mathrm{H}_{5}$ \\
\hline $\mathbf{5 j}$ & $\mathrm{OCH}$ & $\mathrm{CH}_{3}$ & & & \\
\hline
\end{tabular}

Scheme I Preparation of compounds 5a-s. Reagents and conditions: (i) Few drops of concentrated sulfuric acid, absolute methanol, reflux, 4 h; (ii) $\mathrm{H}_{2} \mathrm{~N}-\mathrm{NH}_{2} \bullet \mathrm{H}_{2} \mathrm{O}$, absolute methanol, reflux, $2 \mathrm{~h}$; (iii) Few drops of glacial acetic acid, absolute ethanol, reflux, $4 \mathrm{~h}$. 
Table I Antiproliferative Activities (Cell Growth-Inhibitory Activities at a Concentration of $30 \mu \mathrm{m}$ ) of Compounds 5a-s and Sunitinib Against the Indicated Cell Lines

\begin{tabular}{|l|l|l|l|l|}
\hline Compound No. & HT-29 & ZR-75 & A-549 & Average Growth Inhibition \% \\
\hline $\mathbf{5 a}$ & $75.4 \pm 3.5$ & $57.7 \pm 21.7$ & $90.9 \pm 6.2$ & 80.7 \\
$\mathbf{5 b}$ & $64.5 \pm 6.7$ & $58.9 \pm 13.5$ & $77.0 \pm 9.9$ & 66.8 \\
$\mathbf{5 c}$ & $29.4 \pm 21.4$ & $53.9 \pm 9.5$ & $60.6 \pm 22.3$ & 47.9 \\
$\mathbf{5 d}$ & $13.9 \pm 16.1$ & $40.0 \pm 8.7$ & $6.9 \pm 18.9$ & 20.3 \\
$\mathbf{5 e}$ & $36.5 \pm 8.3$ & $65.9 \pm 8.0$ & $85.3 \pm 11.2$ & 62.6 \\
$\mathbf{5 f}$ & $78.5 \pm 2.8$ & $73.1 \pm 9.8$ & $94.9 \pm 4.7$ & 82.2 \\
$\mathbf{5 g}$ & $95.2 \pm 4.3$ & $90.2 \pm 5.1$ & $97.4 \pm 3.9$ & 94.3 \\
$\mathbf{5 h}$ & $92.4 \pm 5.2$ & $81.8 \pm 8.5$ & $95.8 \pm 4.5$ & 90.0 \\
$\mathbf{5 i}$ & $91.3 \pm 5.4$ & $78.8 \pm 2.3$ & $90.3 \pm 9.3$ & 86.8 \\
$\mathbf{5 j}$ & $90.8 \pm 5.5$ & $82.5 \pm 9.0$ & $97.4 \pm 3.9$ & 90.2 \\
$\mathbf{5 k}$ & $92.7 \pm 2.9$ & $86.6 \pm 3.3$ & $98.4 \pm 2.0$ & 92.6 \\
$\mathbf{5 1}$ & $94.7 \pm 2.9$ & $79.9 \pm 8.8$ & $96.1 \pm 4.6$ & 90.2 \\
$\mathbf{5 m}$ & $94.3 \pm 3.1$ & $83.8 \pm 5.2$ & $99.7 \pm 0.7$ & 92.6 \\
$\mathbf{5 n}$ & $94.3 \pm 1.6$ & $77.5 \pm 8.3$ & $99.1 \pm 1.1$ & 90.3 \\
$\mathbf{5 0}$ & $94.0 \pm 4.8$ & $93.8 \pm 5.1$ & $97.4 \pm 2.9$ & 95.1 \\
$\mathbf{5 p}$ & $93.7 \pm 4.2$ & $89.0 \pm 2.0$ & $96.9 \pm 3.6$ & 93.2 \\
$\mathbf{5 q}$ & $81.6 \pm 5.4$ & $75.4 \pm 12.5$ & $94.6 \pm 2.3$ & 83.9 \\
$\mathbf{5 r}$ & $91.1 \pm 4.2$ & $79.1 \pm 6.4$ & $91.9 \pm 4.6$ & 87.4 \\
$\mathbf{5 s}$ & $97.0 \pm 2.2$ & $92.2 \pm 7.2$ & $98.5 \pm 2.0$ & 95.9 \\
Sunitinib & $59.5 \pm 2.3$ & $90.7 \pm 4.5$ & $85.7 \pm 2.7$ & 78.7 \\
\hline
\end{tabular}

Table 2 The $\mathrm{IC}_{50}$ Values of Compounds 5a-s and Sunitinib Against the Tested Cell Lines

\begin{tabular}{|c|c|c|c|c|}
\hline \multirow[t]{2}{*}{ Compound No. } & \multicolumn{3}{|l|}{$\mathrm{IC}_{50}(\mu \mathrm{M})$} & \multirow[t]{2}{*}{ Average $I C_{50}(\mu M)$} \\
\hline & HT-29 & ZR-75 & A-549 & \\
\hline $5 a$ & $19.74 \pm 1.95$ & $22.73 \pm 5.71$ & $12.98 \pm 2.46$ & 18.48 \\
\hline $5 \mathbf{b}$ & $21.93 \pm 1.29$ & $21.92 \pm 4.93$ & $16.01 \pm 2.26$ & 19.95 \\
\hline $5 c$ & $>30$ & $18.01 \pm 18.47$ & $19.68 \pm 5.20$ & $>22.56$ \\
\hline $5 d$ & $>30$ & $>30$ & $>30$ & $>30$ \\
\hline $5 e$ & $>30$ & $17.87 \pm 7.24$ & $6.68 \pm 1.52$ & 18.18 \\
\hline $5 f$ & $12.88 \pm 1.03$ & $1.53 \pm 31.5$ & $4.04 \pm 0.83$ & 6.15 \\
\hline $5 g$ & $3.68 \pm 0.29$ & $1.01 \pm 3.28$ & $1.49 \pm 0.15$ & 2.06 \\
\hline $5 \mathrm{~h}$ & $5.67 \pm 0.43$ & $0.84 \pm 7.65$ & $2.13 \pm 0.42$ & 2.88 \\
\hline $5 \mathbf{i}$ & $8.92 \pm 0.67$ & $4.31 \pm 7.98$ & $2.82 \pm 0.30$ & 5.35 \\
\hline $5 j$ & $5.73 \pm 0.67$ & $1.48 \pm 7.06$ & $1.93 \pm 0.27$ & 3.05 \\
\hline $5 \mathbf{k}$ & $6.19 \pm 1.58$ & $1.12 \pm 3.05$ & $1.06 \pm 0.37$ & 2.79 \\
\hline 51 & $2.26 \pm 0.33$ & $0.70 \pm 12.04$ & $0.65 \pm 0.12$ & 1.20 \\
\hline $5 \mathrm{~m}$ & $2.02 \pm 0.36$ & $0.74 \pm 0.88$ & $0.76 \pm 0.12$ & 1.17 \\
\hline $5 n$ & $3.50 \pm 0.49$ & $2.10 \pm 3.15$ & $1.41 \pm 0.21$ & 2.34 \\
\hline 50 & $2.28 \pm 0.22$ & $0.86 \pm 0.76$ & $0.64 \pm 0.24$ & 1.26 \\
\hline $5 p$ & $2.64 \pm 0.27$ & $1.30 \pm 0.58$ & $1.05 \pm 0.15$ & 1.66 \\
\hline $5 q$ & $8.11 \pm 1.27$ & $1.51 \pm 5.03$ & $2.13 \pm 0.49$ & 3.92 \\
\hline $5 r$ & $2.45 \pm 0.32$ & $1.28 \pm 12.44$ & $0.64 \pm 0.61$ & 1.46 \\
\hline $5 s$ & $6.34 \pm 0.76$ & $1.32 \pm 2.89$ & $2.41 \pm 0.29$ & 3.36 \\
\hline Sunitinib & $10.14 \pm 0.8$ & $8.31 \pm 2.4$ & $5.87 \pm 0.3$ & 8.11 \\
\hline
\end{tabular}

Abbreviation: $\mathrm{IC}_{50}$, inhibitory concentration $50 \%$. 
$8.11 \mu \mathrm{M})$. Consequently, further pharmacological profiling was conducted with compound $\mathbf{5 m}$ to better understand the potential antiproliferative mechanism of compounds $\mathbf{5 a}-\mathbf{s}$.

\section{Structure Activity Relationship}

The title compounds $\mathbf{5 a} \mathbf{a}-\mathbf{s}$ are structurally classified into five groups namely, $N$-unsubstituted derivatives 5a-e, $N$-methyl derivatives $\mathbf{5 f}-\mathbf{j}, N$-benzyl derivatives $\mathbf{5 k - 0}, N$-4-substituted benzyl derivatives $\mathbf{5 p}-\mathbf{r}$, and $N$-phenyl derivative $\mathbf{5 s}$. In the first group 5a-e, compound 5e, bearing a methoxy substituent in the five position of the isatin nucleus, is the most active congener with an average $\mathrm{IC}_{50}$ value of 18.18 $\mu \mathrm{M}$. Compound $\mathbf{5 g}$, in the second group $\mathbf{5 f}-\mathbf{j}$, have a bromo substituent in the five position of the isatin ring and it showed an average $\mathrm{IC}_{50}$ value of $2.06 \mu \mathrm{M}$ being nearly four times more potent than sunitinib (average $\mathrm{IC}_{50}$ value of $8.11 \mu \mathrm{M}$ ). The third group $\mathbf{5 k}-\mathbf{0}$ seems to be the best group with an average $\mathrm{IC}_{50}$ range of $1.17-2.79 \mu \mathrm{M}$ in which compound $\mathbf{5 m}$, bearing 5-chloroisatin fragment, is the most active congener with an average $\mathrm{IC}_{50}$ value of $1.17 \mu \mathrm{M}$ being about sevenfold more potent than sunitinib. Moreover, the in vitro antiproliferative activity of the fourth group $\mathbf{5 p}-\mathbf{r}$ is in the following order $\mathbf{5 r}>\mathbf{5 p}>\mathbf{5 q}$ with an average $\mathrm{IC}_{50}$ range of 1.46-3.92 $\mu \mathrm{M}$. Compound 5s, bearing $\mathrm{N}$-phenylisatin scaffold displayed good in vitro antiproliferative activity with an average $\mathrm{IC}_{50}$ value of $3.36 \mu \mathrm{M}$ (Table 2). In conclusion, the $N$-benzyl moiety on the isatin residue seems to be the favorable substituent to get good in vitro antiproliferative activity of this class of compounds.

\section{Effects on Cell-Cycle Progression}

The influence of compound $\mathbf{5} \mathbf{m}$ on different aspects of cell-cycle progression was investigated with the A-549 cell line based on the total DNA content of each cell. Additionally, immunofluorescent imaging of the

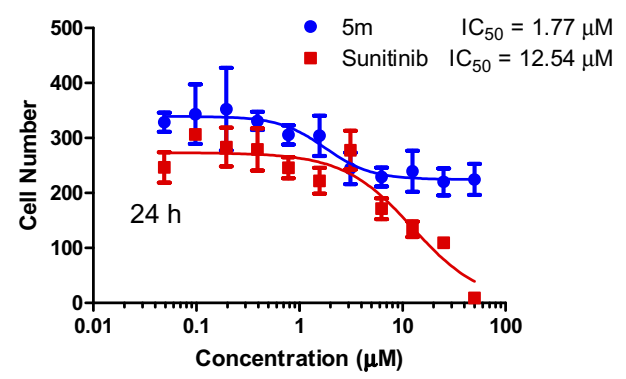

phosphorylated retinoblastoma $(\mathrm{Rb})$ protein was performed over the concentration range, 0.05-100 $\mu \mathrm{M}$. A significant dose-dependent decrease in the total cell number (Figure 2) was noted after treatment with compound $\mathbf{5 m}$ for $48 \mathrm{~h}\left(\mathrm{IC}_{50}\right.$ value $=1.38 \mu \mathrm{M}$; Table 3$)$.

In addition, the percentage of cells in $\mathrm{G} 1$ phase increased and the percentages of cells in the $\mathrm{S}$ and $\mathrm{G} 2 / \mathrm{M}$ phases concomitantly decreased after treatment with compound $\mathbf{5 m}$. This finding suggests that the ability of compound $\mathbf{5 m}$ to influence cell proliferation could be ascribed to decreased cell-cycle progression. Conversely, sunitinib treatment resulted in a reduced percentage of cells in G1 phase and a concomitant increase in cells in $\mathrm{S}$ or $\mathrm{G} 2 / \mathrm{M}$ phase. G2 arrest could be regarded as a checkpoint blockade. Mitotic arrest may, in some instances, result in mitotic catastrophe and subsequent programmed death of cells with multiple or aberrant nuclei.

Moreover, both sunitinib and $\mathbf{5 m}$ caused a substantial dose-dependent decrease in the extent of $\mathrm{Rb}$ protein phosphorylation (Figure 3), suggesting the possibility that the antiproliferative activity of compounds with isatin fragments can be attributed to their effect on cyclindependent kinases. Compound $\mathbf{5 m}$ had an $\mathrm{IC}_{50}$ value of $2.52 \mu \mathrm{M}$ after $24 \mathrm{~h}$, indicating that it was $\sim 1.7$-fold more potent than sunitinib (Table 3 ). Treatment with compound $\mathbf{5 m}$ did not affect the extent of P-Tyr.

\section{Western Blot Analysis}

Data from a previous study revealed a correlation between the antiproliferative marker, B cell translocation gene 1 (BTG1), and the inhibition of proliferation in lung cancer, which suggests that compound $\mathbf{5 m}$ most likely induces cycle arrest during G1 phase. $^{45}$ Treatment with compound $\mathbf{5 m}$ increased BTG1 expression (Figure S1), confirming its potential to act as an antiproliferative agent. To confirm the influence of compound $\mathbf{5 m}$ on cell-cycle progression, the expression levels of different cell cycle-related proteins were examined

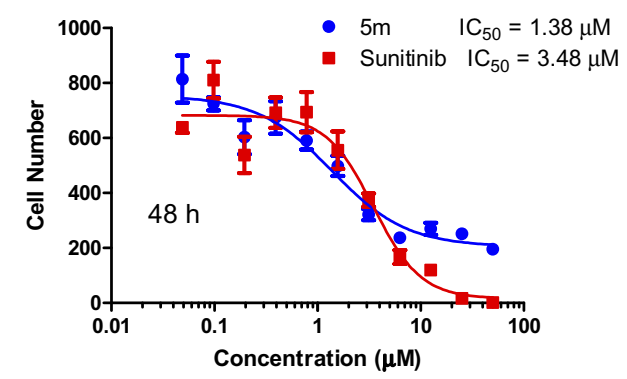

Figure 2 Dose-dependent reductions in the numbers of adherent cells. A-549 NSCLC cells were treated for 24 or 48 h, as indicated, followed by fixation and addition of the fluorescent label. Values are presented as the average number of cells per 4 fields within each well. 
Table $3 \mathrm{IC}_{50}$ Values for the Reductions in Total Cell Numbers and the Effects of Compound $\mathbf{5 m}$ and Sunitinib on Cell-Cycle Progression

\begin{tabular}{|c|c|c|c|c|c|}
\hline \multirow[t]{2}{*}{ Compound No. } & \multicolumn{2}{|c|}{$\begin{array}{l}\text { IC } C_{50}(\mu M) \text { for Reductions in the } \\
\text { Total Cell Numbers }\end{array}$} & \multicolumn{2}{|c|}{$\begin{array}{l}\text { IC } C_{50}(\mu \mathrm{M}) \text { for the Reduction of } \\
\text { Rb Phosphorylation }\end{array}$} & \multirow[t]{2}{*}{ Effects on Cell-Cycle Progression } \\
\hline & $24 \mathrm{~h}$ & $48 \mathrm{~h}$ & $24 \mathrm{~h}$ & $48 \mathrm{~h}$ & \\
\hline $5 \mathrm{~m}$ & $1.77 \pm 1.32$ & $1.38 \pm 0.39$ & $2.52 \pm 0.20$ & $12.15 \pm 3.74$ & GI phase increased, G2/Mphases decreased \\
\hline Sunitinib & $12.54 \pm 9.83$ & $3.48 \pm 0.61$ & $4.37 \pm 0.24$ & $6.27 \pm 0.36$ & GI phase decreased, G2/Mphase increased \\
\hline
\end{tabular}

Abbreviation: $\mathrm{IC}_{50}$, inhibitory concentration $50 \%$.

using Western blot analysis. As depicted in Figure S2, compound $\mathbf{5 m}$ regulated cell cycle-related proteins and activated cyclin-dependent kinase 1 (cdc-2) via phosphorylation at Tyr $15{ }^{46}$ Although cell-cycle analysis revealed an increase in the G1 population after treatment with compound $\mathbf{5 m}$, the ability of $\mathbf{5 m}$ to upregulate the $\mathrm{G} 2 / \mathrm{M}$ phase-related proteins, cyclin B1 and p-cdc2 (Figure S2), was interesting. Ultimately, these findings suggest that cell-cycle arrest after treatment with $\mathbf{5 m}$ can occur during both G1 and G2 phases. ${ }^{47,48}$

Many anticancer agents are designed to cause DNA damage and, consequently, apoptosis. ${ }^{49}$ Hence, the ability of compound $\mathbf{5 m}$ to inhibit cell proliferation by inducing apoptosis was also examined. Compound $\mathbf{5 m}$ activated caspase-3, with increased production of cleaved caspase- 3 within $24 \mathrm{~h}$ of treatment (Figure S3). The expression levels of the prosurvival protein encoded by the B-cell lymphoma/leukemia2 (Bcl-2) gene and the pro-apoptotic protein encoded by the Bcl-2-associated X protein (BAX) gene were also evaluated. Treatment with compound $\mathbf{5 m}$ increase BAX expression but had no obvious effect on Bcl-2 expression (Figure S3). The altered BAX/BCL-2 ratio after treatment with $\mathbf{5 m}$ suggests the involvement of the intrinsic apoptosis pathway.

\section{Selectivity}

The selectivity of compound $\mathbf{5 m}$ was tested using three nontumorigenic cell lines. IEC-6 cells obtained from rat intestines manifested both morphologic and karyotypic properties of normal intestinal epithelial cells. ${ }^{50}$ Cultures obtained from human fibrocystic mammary tissue (MCF10A) are non-tumorigenic and display characteristics similar to primary cultures of breast tissue, including dome formation. ${ }^{51}$ Fibroblasts obtained from the embryonic tissue of mice (Swiss 3 t 3 fibroblasts) are both non-tumorigenic and contact-inhibited. ${ }^{52}$ A-549 NSCLC cells were included for comparison. The antiproliferative activities of compound $\mathbf{5 m}$ and sunitinib were assessed in quadruplicate at a maximum concentration of $25 \mu \mathrm{M}$ and then at 10 serially diluted concentrations. Compound $\mathbf{5 m}$ inhibited cell growth $(>50 \%)$ in both normal and tumor cell lines. However, compound $5 \mathrm{~m}$ exhibited a 2.6-fold selectivity value, whereas sunitinib displayed a 1.4-fold selectivity value (Figure 4 and Table 4).

\section{Multidrug-Resistant Lung Cancer Cell Line}

The antiproliferative activity of compound $\mathbf{5 m}$ was examined against a sensitive NSCLC cell line (A-549) and a multidrugresistant lung cancer cell (NCI-H69AR) expressing the ABCC1 efflux pump protein. Both compound $\mathbf{5 m}$ and sunitinib were tested in quadruplicate at a maximum concentration of $25 \mu \mathrm{M}$, and then at 10 serially diluted concentrations. Compound 5m inhibited growth in both cell lines, with an $\mathrm{IC}_{50}$ value of $1.33 \mu \mathrm{M}$ in A-549 cells (Figure 5 and Table 5).
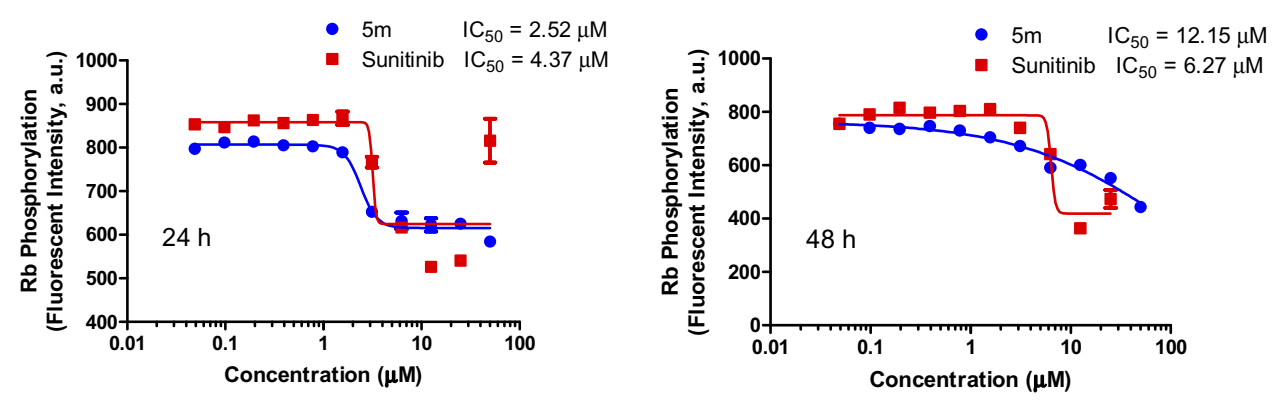

Figure 3 Dose-dependent reductions in Rb phosphorylation. A-549 NSCLC cells were treated for 24 or 48 h (as indicated), fixed, and subjected to quantitative indirect immunofluorescence and image analysis. 

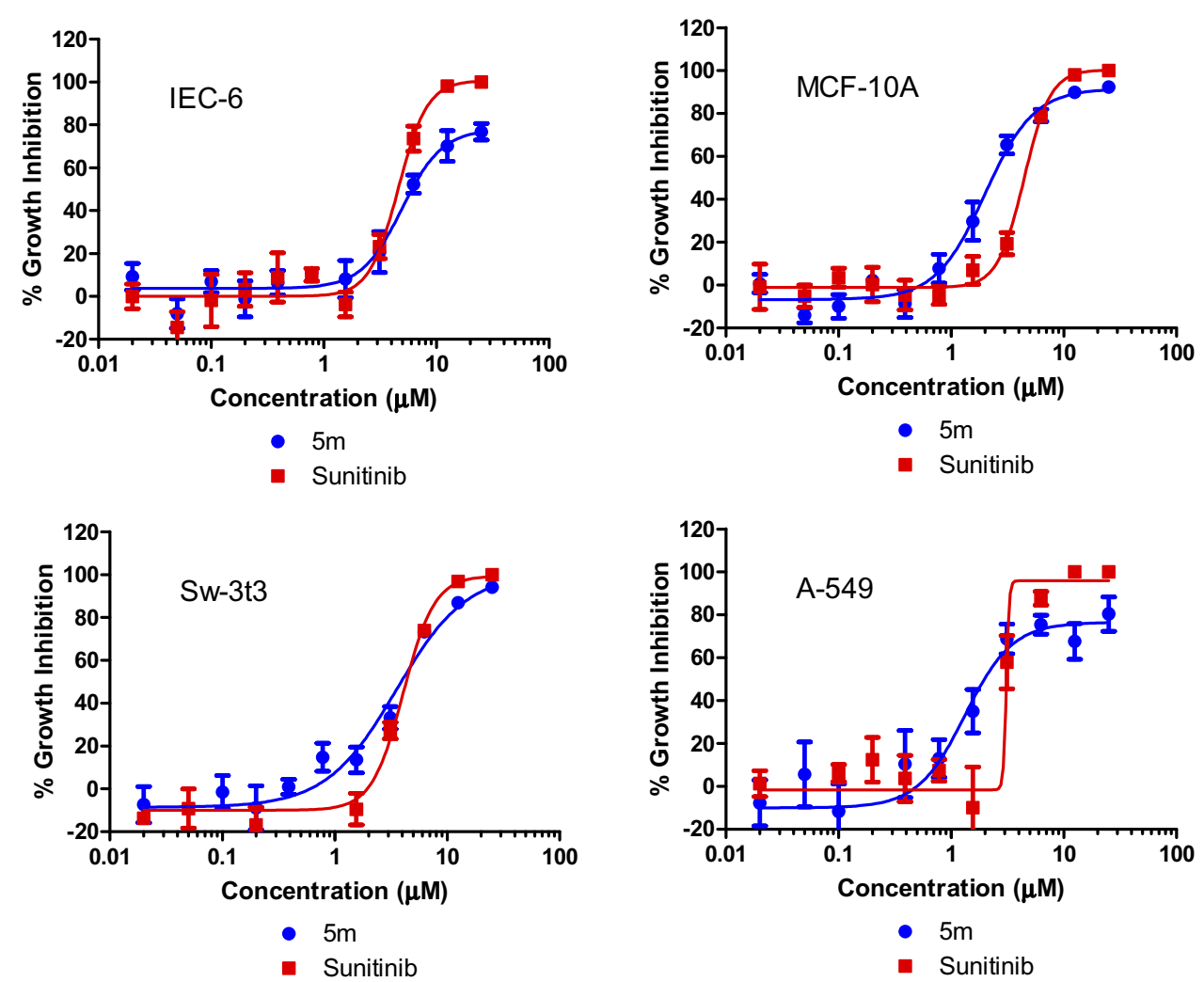

Figure 4 Tumor selectivity. A-549 NSCLC cells and non-tumorigenic cells derived from intestine, breast, and fibroblasts were treated for $72 \mathrm{~h}$, as indicated, for use in a luminescence-based, growth-inhibition assay.

The H69AR cells were $\sim 19$-fold less sensitive, as compound $\mathbf{5 m}$ could be effluxed by the $\mathrm{ABCC} 1$ pump protein. Additionally, a lesser degree of sunitinib resistance (1.9-fold less sensitivity) was found in H69AR cells.

\section{Cell Viability Assay Using HEK 293 Cell Line}

Cell viability assay for compound $\mathbf{5 m}$ showed no apparent toxicity towards human embryonic kidney (HEK) 293 cells following $24 \mathrm{hrs}$ exposure to different $\mathbf{5 m}$ concentrations, indicated by percent viability ranging from 93.72 \pm 0.68 to $98.67 \pm 0.39$. Furthermore, there was no significant change in viability after longer exposure time to $\mathbf{5 m}$ up to 72 hrs.

\section{In Silico Predictions of ADME Properties of Compounds 5a-s}

In silico ADME (absorption, distribution, metabolism and excretion) predictions as well as some pharmacokinetic characteristics of the target compounds $\mathbf{5 a}-\mathbf{s}$ are presented in Table S1 which are calculated according to the literature methods. ${ }^{53,54}$ All the title compounds $\mathbf{5 a}-\mathbf{s}$ follow the Lipinski's rule of 5 (RO5) where $\log P$ values lie between 2.2-4.2 $(<5)$, molecular weight range 304-473 $(<500)$, hydrogen bond acceptor range 3-4 $(<5)$, and hydrogen bond donor range 2-3 $(<5)$, indicating that these compounds might not be expected to cause problems with oral bioavailability. In addition, compound $\mathbf{5 a}-\mathbf{s}$ exhibited topological polar surface area (TPSA) range 77.6-101.4 $\AA^{2}$

Table 4 Selectivity of Compound $\mathbf{5 m}$ and Sunitinib Against Tumor and Non-Tumorigenic Cell Lines

\begin{tabular}{|l|l|l|l|l|l|}
\hline \multirow{2}{*}{ Compound No. } & \multicolumn{2}{|l|}{ IC $_{\mathbf{5 0}}(\boldsymbol{\mu M})$} & \multirow{2}{*}{ Mean Tumor Selectivity } \\
\cline { 2 - 6 } & Intestine IEC-6 & Breast MCF-I 0A & Fibroblast Swiss 3t3 & NSCLC A-549 & \\
\hline 5m & $4.94 \pm 0.82$ & $2.00 \pm 0.24$ & $3.49 \pm 1.14$ & $1.33 \pm 0.47$ & 2.6 \\
Sunitinib & $3.06 \pm 0.54$ & $4.07 \pm 0.23$ & $4.43 \pm 0.75$ & $4.56 \pm \mathrm{amb}$ & 1.4 \\
\hline
\end{tabular}

Abbreviations: $\mathrm{IC}_{50}$, inhibitory concentration $50 \%$; IEC, intestinal epithelial cell; MCF, Michigan cancer foundation; $3 \mathrm{~T} 3$, 3-day transfer, inoculums $3 \times 10^{5}$ cells; NSCLC, nonsmall cell lung cancer. 

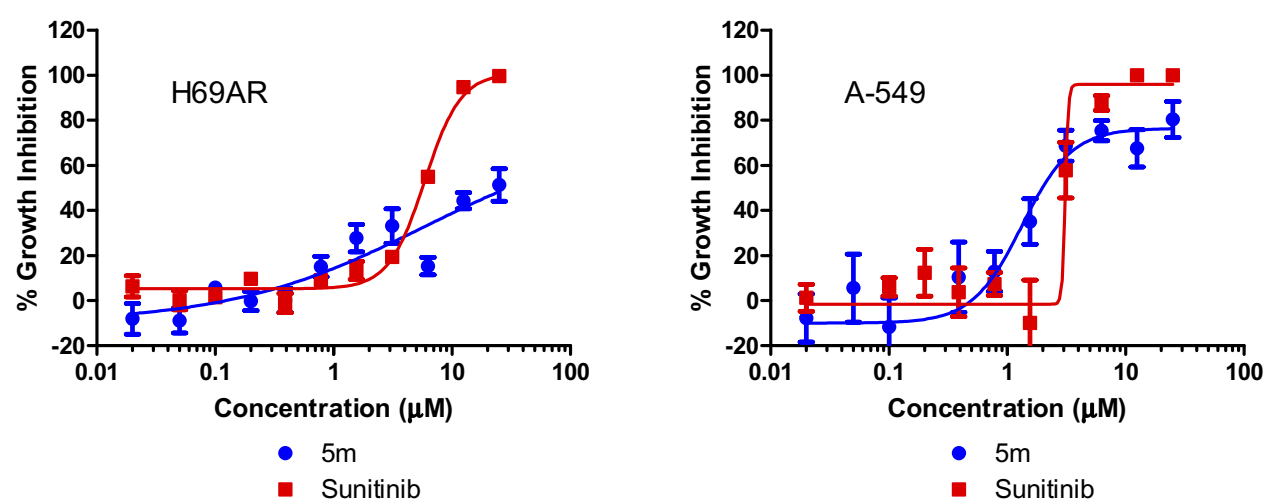

Figure 5 Evaluation of susceptibility to efflux. A-549 NSCLC cells and NCl-H69AR cells expressing the ABCCI (MRPI) multi-drug resistance efflux transporter were treated for $72 \mathrm{~h}$, as indicated, for use in a luminescence-based, growth-inhibition assay.

$\left(<140 \AA^{2}\right)$, denoting good transport through cellular plasma membranes. In summary, compounds $\mathbf{5 a}-\mathbf{s}$ displayed no violations to RO5 criteria suggesting their liability to be new orally bioavailable antiproliferative candidates.

\section{Conclusion}

Herein, we identified the newly synthesized isatin-indole molecular hybrids 5a-s with various spectroscopic approaches and estimated their potentials as antiproliferative agents in vitro. Compound $\mathbf{5 m}$ displayed promising in vitro antiproliferative potential against three human cancer cell lines. With an average $\mathrm{IC}_{50}$ value of 1.17 $\mu \mathrm{M}$, this compound had a seven-fold greater potency than sunitinib $\left(\mathrm{IC}_{50}=8.11 \mu \mathrm{M}\right)$. By deriving its pharmacological profile, we sought to characterize the mechanisms underlying the antiproliferative activities of compounds 5a-s. We found reduced percentages of cells in $\mathrm{S}$ and $\mathrm{G} 2 / \mathrm{M}$ phases, an increased percentage of cells in G1 phase, and a significant dose-dependent decrease in the extent of $\mathrm{Rb}$ protein phosphorylation. Furthermore, Western blot analysis confirmed the extents of BTG1, cyclin B1, cyclin D1, and p-cdc2 upregulation caused by compound $\mathbf{5 m}$. Within $24 \mathrm{~h}$ of cell treatment, compound $\mathbf{5 m}$ increased BAX expression and activated caspase-3, as

Table 5 Antiproliferative Activities of Compound $5 \mathrm{~m}$ and Sunitinib Against Sensitive (a-549) and Resistant (NCl-H69AR) Cell Lines

\begin{tabular}{|l|l|l|l|}
\hline \multirow{2}{*}{$\begin{array}{l}\text { Compound } \\
\text { No. }\end{array}$} & \multicolumn{2}{|l|}{$\mathrm{IC}_{\mathbf{5 0}}(\mu \mathrm{M})$} & $\begin{array}{l}\text { Fold- } \\
\text { Resistance }\end{array}$ \\
\cline { 2 - 3 } & $\begin{array}{l}\text { Sensitive } \\
\text { A-549 }\end{array}$ & $\begin{array}{l}\text { Resistant NCI- } \\
\text { H69AR }\end{array}$ & \\
\hline $\mathbf{5 m}$ & $1.33 \pm 0.47$ & $>25$ & $>19.2$ \\
Sunitinib & 4.56 & $5.8 \pm 0.52$ & 1.9 \\
\hline
\end{tabular}

Abbreviation: $\mathrm{IC}_{50}$, inhibitory concentration $50 \%$. indicated by the observed increase in cleaved caspase- 3 levels. ADME predictions for compounds 5a-s might help in further development of new anticancer agents with favorable oral bioavailability. Based on the findings presented herein, these lead compounds can be utilized in preclinical studies in the field of cancer chemotherapy.

\section{Supporting Materials}

Detailed protocols for the pharmacological evaluation of the title compounds $\mathbf{5 a}-\mathbf{s}$, Western blot results for compound 5m (Figures S1-S3), and ADME Table (Table S1) are provided as Supporting materials.

\section{Acknowledgment}

The authors would like to extend their sincere appreciation to the Deanship of Scientific Research at King Saud University for funding this research through Research Group Project Number RG-1440-140.

\section{Disclosure}

Dr Adam B Keeton reports support of this project through the Deanship of Scientific Research at King Saud University through Research Group Project Number RG-1440-140. The authors declare no other conflicts of interest.

\section{References}

1. Jemal A, Bray F, Center MM, et al. Global cancer statistics. $C A$ Cancer J Clin. 2011;61(2):69-90. doi:10.3322/caac.v61:2

2. Jayashree B, Nigam S, Pai A, et al. Targets in anticancer research-a review. Indian J Exp Biol. 2015;53:489-507.

3. Emami S, Dadashpour S. Current developments of coumarin-based anti-cancer agents in medicinal chemistry. Eur $J$ Med Chem. 2015;102:611-630. doi:10.1016/j.ejmech.2015.08.033

4. Zwick E, Bange J, Ullrich A. Receptor tyrosine kinase signalling as a target for cancer intervention strategies. Endocr Relat Cancer. 2001;8(3):161-173. doi:10.1677/erc.0.0080161 
5. Newman DJ, Cragg GM. Natural products as sources of new drugs over the 30 years from 1981 to 2010. J Nat Prod. 2012;75 (3):311-335. doi:10.1021/np200906s

6. Luo F, Gu J, Chen L, et al. Systems pharmacology strategies for anticancer drug discovery based on natural products. Mol Biosyst. 2014;10(7):1912-1917. doi:10.1039/c4mb00105b

7. Swathi K, Sarangapani M. Synthesis and screening of biologically significant 5 hydroxy isatin derivatives for antioxidant activity. Adv Exp Med Biol. 2015;822:129-137.

8. Sharma PK, Balwani S, Mathur D, et al. Synthesis and anti-inflammatory activity evaluation of novel triazolyl-isatin hybrids. J Enzyme Inhib Med Chem. 2016;31(6):1520-1526. doi:10. 3109/14756366.2016.1151015

9. Gil-Turnes MS, Hay ME, Fenical W. Symbiotic marine bacteria chemically defend crustacean embryos from a pathogenic fungus. Science. 1989;246(4926):116-118. doi:10.1126/science.2781297

10. Vine K, Matesic L, Locke J, et al. Cytotoxic and anticancer activities of isatin and its derivatives: a comprehensive review from 2000-2008. Anticancer Agents Med Chem. 2009;9(4):397-414. doi:10.2174/1871520610909040397

11. Attia MI, Eldehna WM, Afifi SA, et al. New hydrazonoindolin-2-ones: synthesis, exploration of the possible anti-proliferative mechanism of action and encapsulation into PLGA microspheres. PLoS One. 2017;12(7):e0181241. doi:10.1371/ journal.pone.0181241

12. Motzer RJ, Hutson TE, Tomczak P, et al. Sunitinib versus interferon alfa in metastatic renal-cell carcinoma. $N$ Eng $J$ Med. 2007;356 (2):115-124. doi:10.1056/NEJMoa065044

13. Griffith R, Brown M, McCluskey A, et al. Small molecule inhibitors of protein kinases in cancer-how to overcome resistance. Mini Rev Med Chem. 2006;6(10):1101-1110. doi:10.2174/138955706778560 184

14. Garofalo A, Naumova E, Manenti L, et al. The combination of the tyrosine kinase receptor inhibitor SU6668 with paclitaxel affects ascites formation and tumor spread in ovarian carcinoma xenografts growing orthotopically. Clin Cancer Res. 2003;9(9):3476-3485.

15. Krishnegowda G, Gowda AP, Tagaram HRS, et al. Synthesis and biological evaluation of a novel class of isatin analogs as dual inhibitors of tubulin polymerization and Akt pathway. Bioorg Med Chem. 2011;19(20):6006-6014. doi:10.1016/j.bmc.2011.08.044

16. Lakhdar S, Westermaier M, Terrier F, et al. Nucleophilic reactivities of indoles. J Org Chem. 2006;71(24):9088-9095. doi:10.1021/ jo0614339

17. Diss LB, Robinson SD, Wu Y, et al. Age-related changes in melatonin release in the murine distal colon. ACS Chem Neurosci. 2013;4 (5):879-887. doi:10.1021/cn4000617

18. de Sa A, Fernando R, Barreiro EJ, et al. From nature to drug discovery: the indole scaffold as a 'privileged structure'. Mini Rev Med Chem. 2009;9(7):782-793. doi:10.2174/138955709788 452649

19. Ahmad A, A Sakr W, Wahidur Rahman K. Anticancer properties of indole compounds: mechanism of apoptosis induction androle in chemotherapy. Curr Drug Targets. 2010;11(6):652-666. doi:10.21 74/138945010791170923

20. Akkoç MK, Yüksel MY, Durmaz İ, et al. Design, synthesis, and biological evaluation of indole-based 1, 4-disubstituted piperazines as cytotoxic agents. Turk J Chem. 2012;36(4):515-525.

21. MacDonough MT, Strecker TE, Hamel E, et al. Synthesis and biological evaluation of indole-based, anti-cancer agents inspired by the vascular disrupting agent 2-(3'-hydroxy-4'-methoxyphenyl) -3-(3",4",5"-trimethoxybenzoyl)-6-methoxyindole (OXi8006). Bioorg Med Chem. 2013;21(21):6831-6843. doi:10.1016/j.bmc.2013.07.028

22. Almagro L, Fernández-Pérez F, Pedreño M. Indole alkaloids from catharanthus roseus: bioproduction and their effect on human health. Molecules. 2015;20(2):2973-3000. doi:10.3390/molecules 20022973
23. Bradner W. Mitomycin C: a clinical update. Cancer Treat Rev. 2001;27(1):35-50. doi:10.1053/ctrv.2000.0202

24. Singh Sidhu J, Singla R, Jaitak V, Jaitak V. Indole derivatives as anticancer agents for breast cancer therapy: a review. Anticancer Agents Med Chem. 2016;16(2):160-173. doi:10.2174/1871520615 666150520144217

25. Sravanthi T, Manju S. Indoles-a promising scaffold for drug development. Eur J Pharm Sci. 2016;91:1-10. doi:10.1016/j. ejps.2016.05.025

26. Chadha N, Silakari O. Indoles as therapeutics of interest in medicinal chemistry: bird's eye view. Eur J Med Chem. 2017;134:159-184. doi:10.1016/j.ejmech.2017.04.003

27. Almutairi MS, Hassan ES, Keeton AB, et al. Antiproliferative activity and possible mechanism of action of certain 5-methoxyindole tethered C-5 functionalized isatins. Drug Des Devel Ther. 2019;13:3069. doi:10.2147/DDDT

28. Sang Y-L, Zhang W-M, Lv P-C, et al. Indole-based, antiproliferative agents targeting tubulin polymerization. Curr Top Med Chem. 2016;17(2):120-137. doi:10.2174/1568026616666160530154812

29. Song Y-L, Dong Y-F, Yang T, et al. Synthesis and pharmacological evaluation of novel bisindolylalkanes analogues. Bioorg Med Chem. 2013;21(24):7624-7627. doi:10.1016/j.bmc.2013.10.034

30. Giannini G, Marzi M, Di Marzo M, et al. Exploring bis-(indolyl) methane moiety as an alternative and innovative CAP group in the design of histone deacetylase (HDAC) inhibitors. Bioorg Med Chem Lett. 2009;19(10):2840-2843. doi:10.1016/j.bmcl.2009.03.101

31. Haddach M, Michaux J, Schwaebe MK, et al. Discovery of CX-6258. A potent, selective, and orally efficacious pan-pim kinases inhibitor. ACS Med Chem Lett. 2012;3(2):135-139. doi:10.1021/ml200259q

32. Xia Y, Fan C-D, Zhao B-X, et al. Synthesis and structure-activity relationships of novel 1-arylmethyl-3-aryl-1H-pyrazole-5-carbohydrazide hydrazone derivatives as potential agents against A549 lung cancer cells. Eur J Med Chem. 2008;43:2347-2353. doi:10.1016/j. ejmech.2008.01.021

33. Zhang D, Wang G, Zhao G, et al. Synthesis and cytotoxic activity of novel 3-(1H-indol-3-yl)-1H-pyrazole-5-carbohydrazide derivatives. Eur J Med Chem. 2011;46:5868-5877. doi:10.1016/j.ejmech.2011. 09.049

34. Pieczonka AM, Strzelczyk A, Sadowska B, et al. Synthesis and evaluation of antimicrobial activity of hydrazones derived from 3-oxido-1H-imidazole-4-carbohydrazides. Eur J Med Chem. 2013;64:389-395. doi:10.1016/j.ejmech.2013.04.023

35. Gediya LK, Njar VC. Promise and challenges in drug discovery and development of hybrid anticancer drugs. Expert Opin Drug Discov. 2009;4(11):1099-1111. doi:10.1517/17460440903341705

36. Mishra S, Singh P, Singh P. Hybrid molecules: the privileged scaffolds for various pharmaceuticals. Eur J Med Chem. 2016;124:500-536. doi:10.1016/j.ejmech.2016.08.039

37. Kerru N, Singh P, Koorbanally N, et al. Recent advances (2015-2016) in anticancer hybrids. Eur J Med Chem. 2017;142:179-212. doi:10.1016/j.ejmech.2017.07.033

38. Al-Wabli R, Zakaria A, Attia M. Synthesis, spectroscopic characterization and antimicrobial potential of certain new isatin-indole molecular hybrids. Molecules. 2017;22(11):1958. doi:10.3390/molecules 22111958

39. Abdel-Aziz HA, Eldehna WM, Keeton AB, et al. Isatin-benzoazine molecular hybrids as potential antiproliferative agents: synthesis and in vitro pharmacological profiling. Drug Des Devel Ther. 2017; 11:2333. doi:10.2147/DDDT

40. Grauzdyte D, Pukalskas A, Viranaicken W, et al. Protective effects of phyllanthus phillyreifolius extracts against hydrogen peroxide induced oxidative stress in HEK293 cells. PLoS One. 2018;13(11): e0207672. doi:10.1371/journal.pone.0207672

41. Almutairi MS, Ghabbour HA, Attia MI. Crystal structure of methyl $1 \mathrm{H}$-indole-2-carboxylate, $\mathrm{C}_{10} \mathrm{H}_{9} \mathrm{NO}_{2}$. Z Krist-New Cryst St. 2017;232 (3):431-432. 
42. Almutairi M, Zakaria A, Al-Wabli R, et al. Synthesis, spectroscopic identification and molecular docking of certain $N$-(2-\{[2-(1H-indol-2-ylcarbonyl)hydrazinyl] (oxo)acetyl $\}$ phenyl) acetamides and $N$-[2-(2-\{[2-(acetylamino)phenyl](oxo) acetyl $\}$ hydrazinyl)-2-oxoethyl]-1 $H$-indole-2-carboxamides: new antimicrobial agents. Molecules. 2018;23(5):1043.

43. Haress NG, Ghabbour HA, Almutairi MS, et al. Crystal structure of 5-methoxy- $N^{\prime}$-[(3Z)-5-chloro-1-(4-fluorobenzyl)-2-oxo-1,2-dihydro$3 H$-indol-3-ylidene]-1 $H$-indole-2-carbohydrazide-DMSO (1/1), $\mathrm{C}_{25}$ $\mathrm{H}_{18} \mathrm{ClFN}_{4} \mathrm{O}_{3} \cdot \mathrm{C}_{2} \mathrm{H}_{6} \mathrm{OS} . \quad Z$ Krist-New Cryst St. 2016;231(4):10 21-1023.

44. Almutairi MS, Ghabbour HA, Haress NG, et al. Crystal structure of 5-methoxy- $N^{\prime}$-[(3Z)-1-benzyl-5-fluoro-2-oxo-1,2-dihydro-3 $\mathrm{H}$-indol3-ylidene]-1 $H$-indole-2-carbohydrazide-DMSO (1/1), $\mathrm{C}_{27} \mathrm{H}_{25} \mathrm{FN}_{4} \mathrm{O}_{4}$ S. Z Krist-New Cryst St. 2016;231(4):1025-1027.

45. Sun G, Lu Y, Cheng Y, et al. The expression of BTG1is downregulated in NSCLC and possibly associated with tumor metastasis. Tumor Biol. 2014;35(4):2949-2957. doi:10.1007/s13277-013-1379-6

46. Welburn JP, Tucker JA, Johnson T, et al. How tyrosine 15 phosphorylation inhibits the activity of cyclin-dependent kinase 2-cyclin A. J Biol Chem. 2007;282(5):3173-3181. doi:10.1074/jbc.M609151200

47. Blajeski AL, Phan VA, Kottke TJ, et al. G 1 and G 2 cell-cycle arrest following microtubule depolymerization in human breast cancer cells. J Clin Invest. 2002;110(1):91-99. doi:10.1172/JCI13275
48. Liu X, Zhao P, Wang X, et al. Celastrol mediates autophagy and apoptosis via the ROS/JNK and Akt/mTOR signaling pathways in glioma cells. J Exp Clin Cancer Res. 2019;38(1):184. doi:10.1186/ s13046-019-1173-4

49. Rixe O, Fojo T. Is cell death a critical end point for anticancer therapies or is cytostasis sufficient? Clin Cancer Res. 2007;13 (24):7280-7287. doi:10.1158/1078-0432.CCR-07-2141

50. Quaroni A, Wands J, Trelstad RL, et al. Epithelioid cell cultures from rat small intestine. Characterization by morphologic and immunologic criteria. J Cell Biol. 1979;80(2):248-265. doi:10.1083/jcb.80.2.248

51. Soule HD, Maloney TM, Wolman SR, et al. Isolation and characterization of a spontaneously immortalized human breast epithelial cell line, MCF-10. Cancer Res. 1990;50(18):6075-6086.

52. Todaro GJ, Green H. Quantitative studies of the growth of mouse embryo cells in culture and their development into established lines. J Cell Biol. 1963;17:299-313. doi:10.1083/jcb.17.2.299

53. Kovačević SZ, Jevrić LR, Kuzmanović SOP, et al. Prediction of in-silico ADME properties of 1,2-O-isopropylidene aldohexose derivatives. Iran J Pharm Res. 2014;13(3):899-907.

54. Daina A, Michielin O, Zoete V. SwissADME: a free web tool to evaluate pharmacokinetics, drug-likeness and medicinal chemistry friendliness of small molecules. Sci Rep. 2017;7:42717. doi:10. $1038 /$ srep42717

\section{Publish your work in this journal}

Drug Design, Development and Therapy is an international, peerreviewed open-access journal that spans the spectrum of drug design and development through to clinical applications. Clinical outcomes, patient safety, and programs for the development and effective, safe, and sustained use of medicines are a feature of the journal, which has also been accepted for indexing on PubMed Central. The manuscript management system is completely online and includes a very quick and fair peer-review system, which is all easy to use. Visit http://www. dovepress.com/testimonials.php to read real quotes from published authors. 